\title{
Dissecting the control of shoot development in grapevine: genetics and genomics identify potential regulators
}

\author{
Sabine Guillaumie ${ }^{1 *} \mathbb{D}$, Stéphane Decroocq ${ }^{2}$, Nathalie Ollat ${ }^{1}$, Serge Delrot ${ }^{1}$, Eric Gomès ${ }^{1 \dagger}$ and Sarah J. Cookson ${ }^{1 \dagger}$
}

\begin{abstract}
Background: Grapevine is a crop of major economic importance, yet little is known about the regulation of shoot development in grapevine or other perennial fruits crops. Here we combine genetic and genomic tools to identify candidate genes regulating shoot development in Vitis spp.

Results: An F2 population from an interspecific cross between $V$. vinifera and $V$. riparia was phenotyped for shoot development traits, and three Quantitative Trait Loci (QTLs) were identified on linkage groups (LGs) 7, 14 and 18. Around $17 \%$ of the individuals exhibited a dwarfed phenotype. A transcriptomic study identified four candidate genes that were not expressed in dwarfed individuals and located within the confidence interval of the QTL on LG7. A deletion of 84,482 bp was identified in the genome of dwarfed plants, which included these four not expressed genes. One of these genes was VVICURLY LEAF (VViCLF), an orthologue of CLF, a regulator of shoot development in Arabidopsis thaliana.
\end{abstract}

Conclusions: The phenotype of the dwarfed grapevine plants was similar to that of clf mutants of $A$. thaliana and orthologues of the known targets of CLF in A. thaliana were differentially expressed in the dwarfed plants. This suggests that CLF, a major developmental regulator in A. thaliana, also controls shoot development in grapevine.

Keywords: CURLY LEAF, Dwarfed phenotype, F2 population, Quantitative trait loci, Shoot development, Vitis interspecific cross

\section{Background}

Grapevine (Vitis spp.) is one of the most economically important perennial fruit crops cultivated worldwide and the regulation of shoot development is central to fruit yield and berry oenological potential. On a botanical basis, grapevine is a liana with indeterminate growth habits. Commercially grown grapevines produce annual shoots that emerge from a given number of latent buds retained after pruning and shoot development results from the recurrent production and development of phytomers. Like most deciduous woody plants, annual shoot development in grapevine begins with the development of latent buds containing generally six to 10 pre-formed

\footnotetext{
* Correspondence: sabine.guillaumie@inrae.fr

${ }^{\dagger}$ Eric Gomès and Sarah J. Cookson contributed equally to this work.

'UMR1287 EGFV, Bordeaux Sciences Agro, INRAE, University of Bordeaux,

Villenave d'Ornon, France

Full list of author information is available at the end of the article
}

phytomers, followed by the indeterminate production of neo-formed phytomers [1].

The molecular control of shoot development has been well characterised in model species such as Arabidopsis thaliana, through the identification and characterisation of mutants and gene networks underlying different shoot developmental phenotypes [2]. In grapevine ( $V$. vinifera L.), only a small number of shoot development mutants have been identified in grapevine. One example is the Vvigail dwarf gibberellin insensitive mutant derived from the L1 layer of $V$. vinifera cv. Pinot meunier, which produces extremely short internodes and inflorescences in the place of tendrils along the shoot [3]. Other dwarf grapevines have been identified based on gibberellin insensitivity $[4,5]$. These dwarf grapevines are of particular interest for genetic studies in small controlled environments [4].

In addition to gibberellin insensitive mutants described above, dwarf phenotypes have been reported with a high frequency within self-progenies of $V$. vinifera and $V$. 
riparia hybrids and other interspecific progenies [6, 7]. The underlying mechanisms and the genetic architecture of such abnormalities are not known. However, cultivated, clonally propagated grapevines are known to present a high level of heterozygosity and may carry a heavy load of deleterious recessive alleles; as such they are highly susceptible to inbreeding depression [8]. Plants presenting inbreeding depression symptoms offer the opportunity to understand plant functioning via the identification of the loci and/or the molecular mechanisms potentially involved.

Quantitative Trait Loci (QTL) analysis has been widely used to describe the genetic architecture of phenotypic traits segregating in interspecific and intraspecific crosses. For example, QTLs have been identified for various shoot development related traits in grapevine, particularly those associated with leaf area [5], inflorescence morphology [9], berry development and composition such as weight, colour, sugar or acid contents $[5,10,11]$ and phenology [12-15]. To date, the genetic architecture of internode length in grapevine has only been studied on a cross between the Picovine 00C001V0008 (Vvigai1/Vvigail, a dwarf vine with a rapid life cycle) and the $V$. vinifera $\mathrm{cv}$. Ugni Blanc fleshless berry mutant [5]. No QTLs repeated over years were found for this trait. Further studies are therefore required to provide new insights into the genetic control of this trait. Screening parents for dwarfism with genetic makers could help to develop efficient breeding programs.

Most QTL studies in grapevine have been performed on F1 populations [16] and only four grapevine genetic maps based on F2 populations have been published [11, 17-19]. Compared to an F1-based mapping strategy, genetic maps developed on F2 populations (with at least 200 progeny) have superior linkage map accuracy and enable the capture of additional meiotic events and recessive allele effects such as those potentially underlying inbreeding depression symptoms [11, 20]. However, previous F2 studies in grapevine have not yet characterised the control of dwarfing traits.

In the present study, we combined QTL mapping with transcriptomics to identify the potential regulators of shoot development in grapevine. The genetic architecture of shoot development was characterised in an F2 population derived from a cross between $V$. vinifera and V. riparia. Approximately $17 \%$ of this population presented dwarfed phenotypes. The transcripts differentially expressed in the young shoots of plants with dwarfed and normal phenotypes were compared using oligonucleotide microarrays. This led to the identification of a deletion in the genome of $V$. vinifera cv. CabernetSauvignon (CS) which, based on the homology with genes from $A$. thaliana, contains potential shoot development regulators.

\section{Results}

\section{Genetic linkage map construction}

For linkage mapping, a total of 173 simple sequence repeat (SSR) markers were tested with DNA samples from CS, $V$. riparia cv. Gloire de Montpellier (RGM), F1_148 and five individuals of the CS x RGM_F2 population. Among them, 47 new markers were developed for this study (Additional file 1). Four SSR markers had a monomorphic profile (VVMD25, UDV013, VMC3C11, VVBX07), five had a complex or multiloci profile (VVBX13, VVIH02, VVIM72, VVIV70, UDV061) and 18 were not reliably amplified, and were all removed from the analysis.

The remaining 146 polymorphic markers were used to genotype the 337 individuals of the CS x RGM_F2 population and to construct and validate the genetic linkage map by the use of the softwares Carthagene and JoinMap 3.0 (Additional file 2). Only the VMC2A9 and VVIP17 markers related to multiple loci were kept. When multiple loci were amplified with the same primer pair, the suffix ' $a$ ' ' $b$ ' or 'CS' 'RGM' was added to the marker name (Additional file 2). The average number of individuals genotyped per loci was 336 with a minimum of 325 individuals genotyped.

All the 146 markers were linked and mapped into 19 LG. The total length of the map was 1051.1 centiMorgan (cM) with an average distance of $7.2 \mathrm{cM}$ between markers and 7.68 markers per LG (Additional file 3). Ten gaps larger than $20 \mathrm{cM}$ were identified. The largest gap, between markers VVC34 and VVIP26 on LG 14, was $30.3 \mathrm{cM}$. Linkage group sizes ranged from $47.4 \mathrm{cM}$ (LG5) to $76.2 \mathrm{cM}$ (LG18) with an average size of 55.3 $\mathrm{cM}$. The marker order was consistent with the order determined from the F1 population CS x RGM1995-1 and from the $V$. vinifera $12 \mathrm{X}$ genome sequence.

\section{QTL detection of shoot development traits}

To detect QTLs, the winter cane pruning weight (CPW) and internode length (IL) of 326 genotypes of the F2 population were measured. The population showed considerable phenotypic variation for each trait (Fig. 1). They did not display a normal distribution and appeared to be made up of two populations of different sizes, normal and dwarfed. Seventeen percent of the CS X RGM F2 population was composed of dwarfed plants, defined in this study as having a winter CPW of less than $25 \mathrm{~g}$. In addition, both stem development, and leaf shape and size were affected (Fig. 2; Additional file 4). The dwarfed plants had curled-leaf phenotype (Fig. 2d and Additional file $4 \mathrm{e}-\mathrm{g}$ ) and no flowers.

Pearson correlation coefficients were calculated for each IL and CPW between years and the two traits during a single year $(p<0.05)$. For each correlation tested, a significant positive coefficient was found. Highly significant correlations were found both for each trait between 


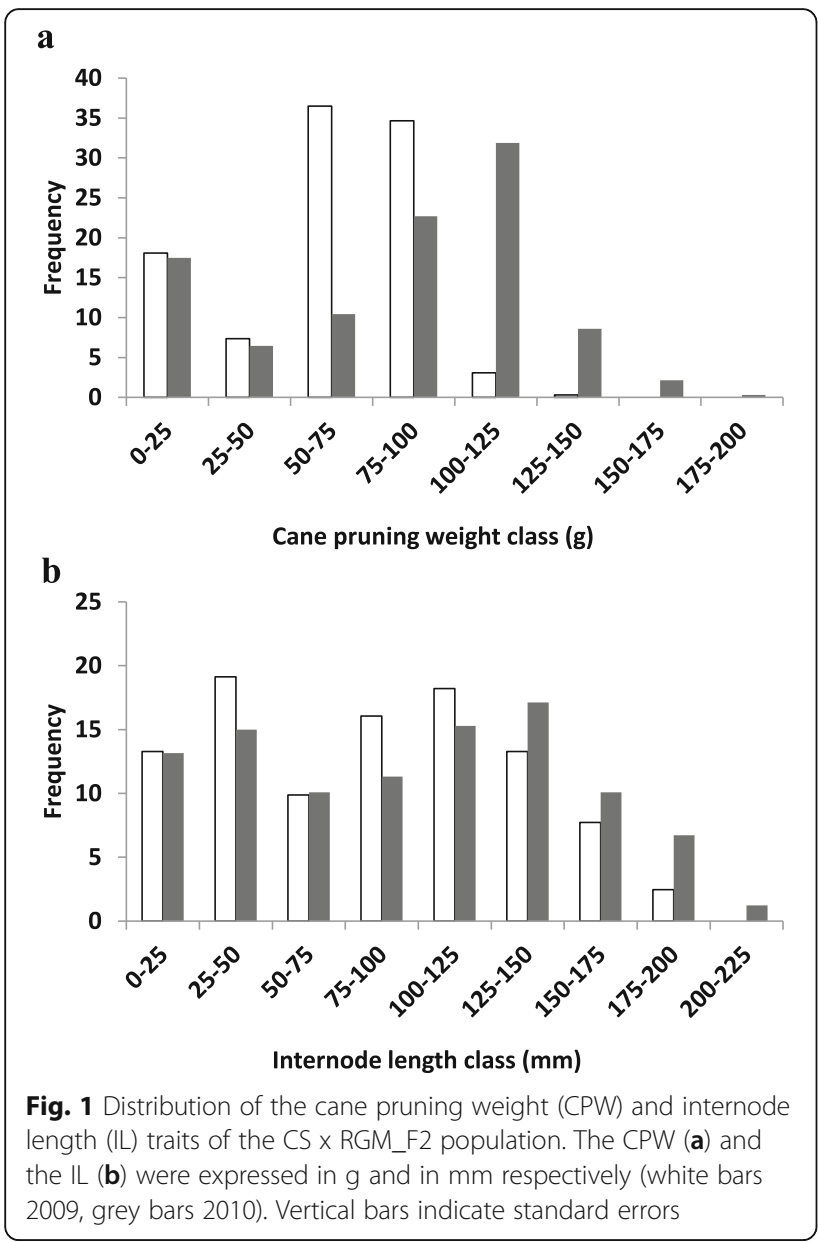

years $\left(\mathrm{r}^{2}=0.80\right.$ and 0.86 for winter CPW and IL respectively), and for the different traits in each year $\left(r^{2}=0.52\right.$ and 0.64 for 2009 and 2010 respectively).

The non-parametric Kruskal-Wallis test suggested the existence of QTLs for CPW and IL on LG7, LG14 and LG18 (Table 1). Then, the Multiple QTL Mapping (MQM) analysis identified 10 significant QTLs of shoot development traits with a significant LOD score $>3.0$. Four QTLs were located on LG7 and LG18 for CPW and six were located on LG7, LG14 and LG18 for IL (Table 1; Fig. 3). QTLs detected on LG7 and LG18 for CPW colocated with QTLs for IL detected on same LGs.

For CPW and IL traits, the major QTL was detected on LG7 and explained in 2009 between 27.1 and 49.8\% of the phenotypic variance. A second QTL was also identified for both CPW and IL on LG18 and explained in 2009 from 2.9 to $13.7 \%$ of the phenotypic variance. An additional QTL explaining 12.9\% in 2009 and $14.8 \%$ in 2010 of the phenotypic variance was detected for IL trait and located on LG14.

At the VVIV04 closest locus of major QTLs peaks on the LG7, an 'aa' genotype indicated a negative impact of the CS allele ' $a$ ' on shoot development when this allele is

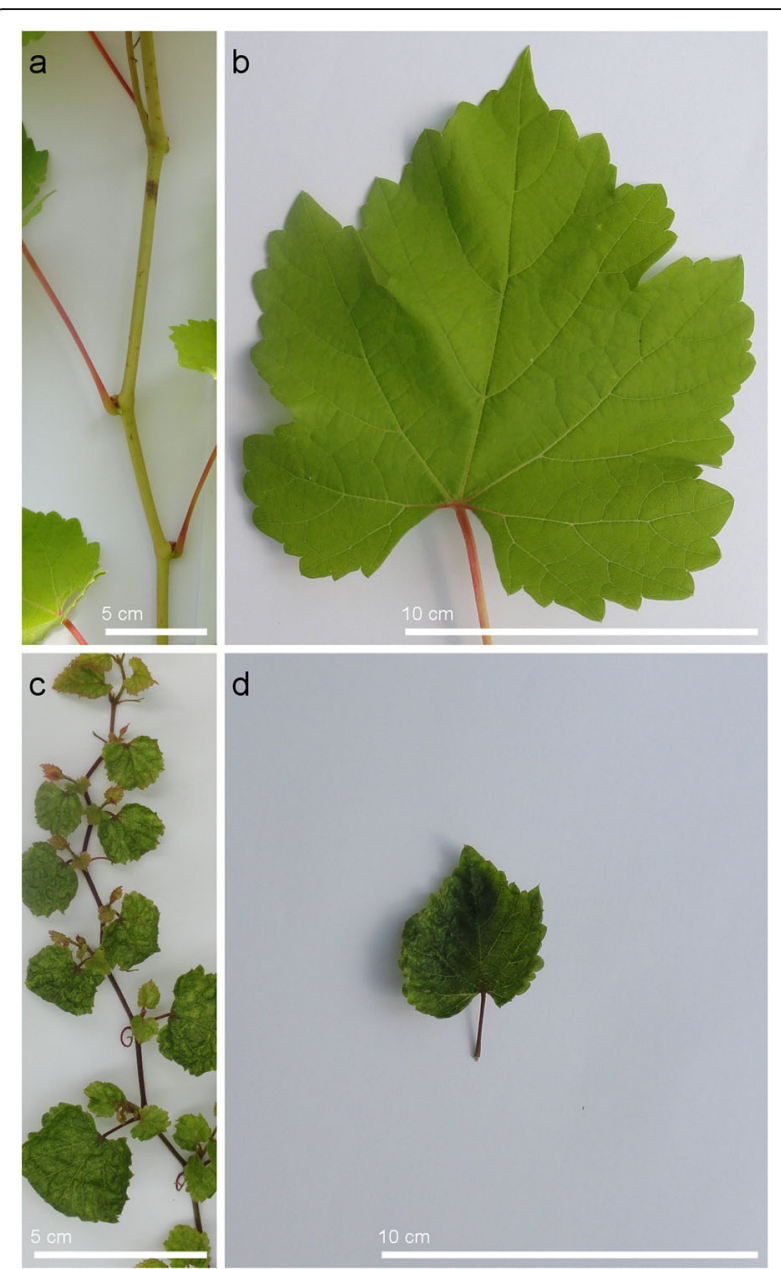

Fig. 2 Leaf and internode phenotypes. a and b "Normal" internode and leaf phenotype of F2_259 genotype. $\mathbf{c}$ and $\mathbf{d}$ Dwarfed shoot and leaf of F2_024 plant. Bars: $5 \mathrm{~cm}(\mathbf{a}, \mathbf{c}), 10 \mathrm{~cm}(\mathbf{b}, \mathbf{d})$

homozygous $(P<0.0001)$ (Fig. 4). The 'aa' genotype was also deleterious for CPW and IL at the other loci $(P<0.001)$ except for CPW at the VVIN94 locus $(P=0.49$ in 2009, $P=$ 0.51 in 2010) (Fig. 3 c-f).

Based on the physical map, a total of 250, 122 and 787 genes were located within the flanking markers of the \pm 2-LOD confidence intervals of QTLs on LG7, LG14 and LG18 respectively.

\section{Genes differentially expressed between the dwarfed and normal individuals}

The transcriptomes of young shoots (leaves and stems) of five normal and five dwarfed plants of the population CSxRGM_F2 were analysed using whole genome microarrays and the abundance of six transcripts was confirmed by qPCR (Additional file 5). Forty-four transcripts were up-regulated, and 8 transcripts were down-regulated in the dwarfed plants (log2 fold change $>1, p<0.05$ adjusted with the Holm method) (Table 2). 
Table 1 Parameters associated with quantitative trait loci (QTLs) detected by multiple QTL mapping (MQM) for vegetative variables measured on the CSxRGM_F2 progeny

\begin{tabular}{|c|c|c|c|c|c|c|c|c|c|c|c|}
\hline Trait & Year & LG & $\begin{array}{l}\text { Position } \\
\text { (cM) }\end{array}$ & Locus & LOD & $\begin{array}{l}\text { LOD threshold } a=0.05 \% \text { on } \\
\text { the linkage group }\end{array}$ & $\begin{array}{l}\text { LOD threshold } a=0.05 \% \text { on } \\
\text { the whole genome }\end{array}$ & $\begin{array}{l}\text { Confidence interval } \pm 2- \\
\text { LOD (cM) }\end{array}$ & $R^{2}$ & $\begin{array}{l}\text { Global } \\
R^{2}\end{array}$ & $\mathrm{KW}$ \\
\hline CPW & 2009 & 7 & 55.5 & WIV04 & 53.07 & 3.2 & 4.7 & 53.3-57 & 0.498 & 0.518 & $* * * *$ \\
\hline CPW & 2009 & 18 & 9.2 & VMC8B5 & 4.18 & 3.5 & & $2.9-15.9$ & 0.029 & & $* * * *$ \\
\hline CPW & 2010 & 7 & 55.5 & WIV04 & 50.13 & 3.4 & 4.5 & $53-57$ & 0.446 & 0.537 & $* * * *$ \\
\hline CPW & 2010 & 18 & 9.2 & VMC8B5 & 12.09 & 3.5 & & $4.5-13.6$ & 0.086 & & $* * * *$ \\
\hline IL & 2009 & 7 & 55.5 & WIV04 & 36.81 & 3.2 & 4.8 & $52.8-56.8$ & 0.271 & 0.565 & $* * * *$ \\
\hline IL & 2009 & 14 & 62.1 & WIN94 & 20.27 & 3.2 & & $59.1-64.3$ & 0.129 & & $* * * *$ \\
\hline IL & 2009 & 18 & 9.2 & VMC8B5 & 19.41 & 3.5 & & $5.8-12$ & 0.137 & & $* * * *$ \\
\hline IL & 2010 & 7 & 55.5 & WIV04 & 46.33 & 3.0 & 4.6 & $52.7-59.6$ & 0.330 & 0.625 & $* * * *$ \\
\hline IL & 2010 & 14 & 62.1 & WIN94 & 25.12 & 3.3 & & $59.3-63.8$ & 0.148 & & $* * * *$ \\
\hline IL & 2010 & 18 & 9.2 & VMC8B5 & 19.92 & 3.3 & & $5.8-12.2$ & 0.122 & & $* * * *$ \\
\hline
\end{tabular}

For each trait is described, the linkage group (LG) where the QTL was identified, position on the map, name of the closest locus to the logarithm of the odds (LOD) peak, LOD value, LOD threshold on the linkage group and on the whole genome with $a=0.05 \%$, confidence interval, phenotypic variance explained by the QTL $\left(R^{2}\right)$, global variance explained by all the QTLs detected for one trait (Global $R^{2}$ ), and significant degree according to non-parametric Kruskal-Wallis test (KM) CPW Cane pruning weight; $I L$ internode length. Statistical significance: ${ }^{* * *}, p<0.0001$

\section{LG7}

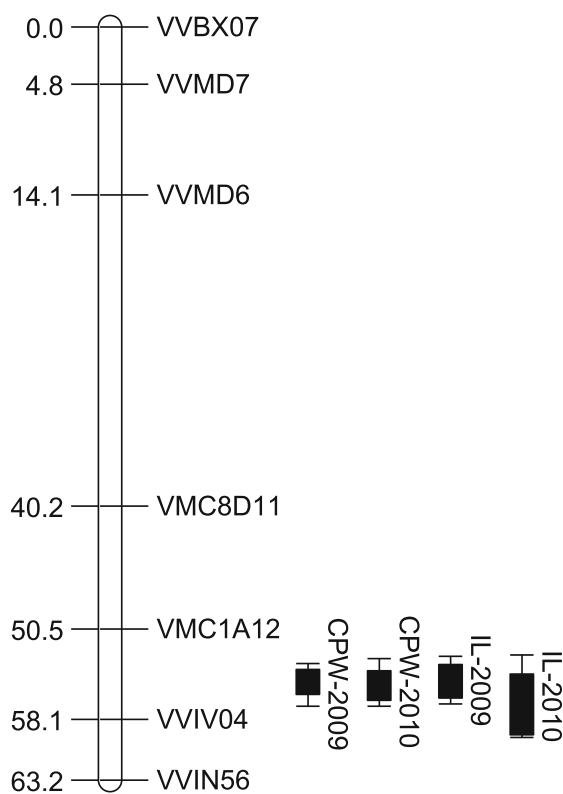

LG14

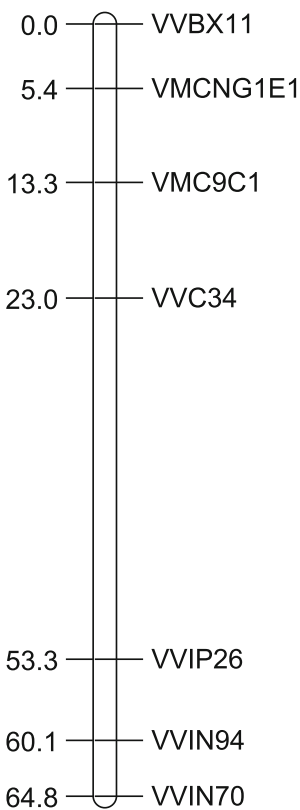

\section{LG18}

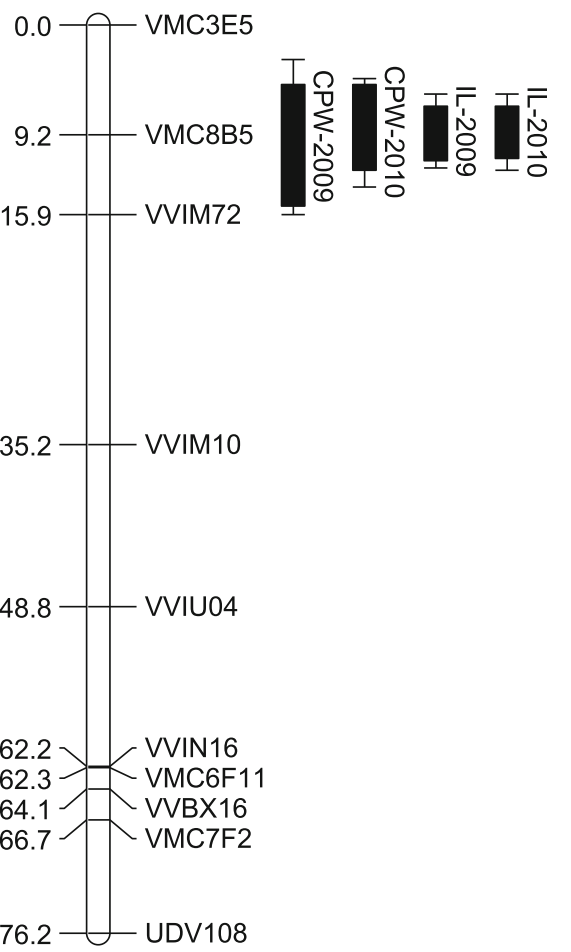

Fig. 3 Genomic position of quantitative trait loci (QTLs) detected on the linkage groups of the CS X RGM_F2 map by multiple QTL mapping (MQM). QTLs are represented by boxes extended by lines representing the logarithm of the odds (LOD)-1 and LOD-2 confidence intervals. Linkage groups are named according to international consensus map. CPW, cane pruning weight; LG, linkage group; IL, internode length. Distances are in CM Kosambi 
a

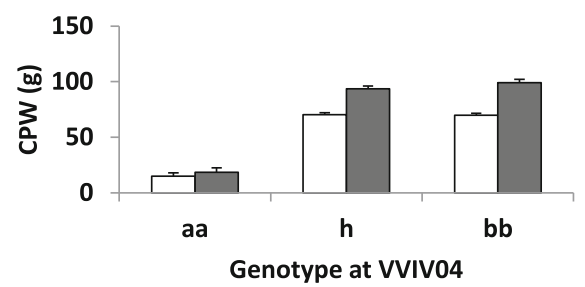

C

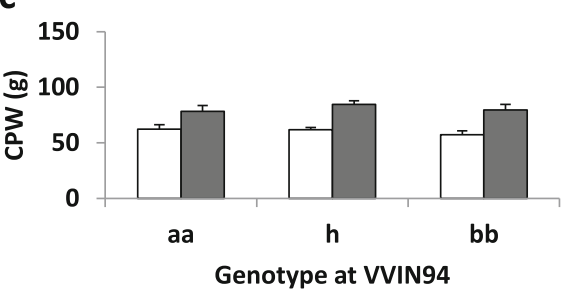

e

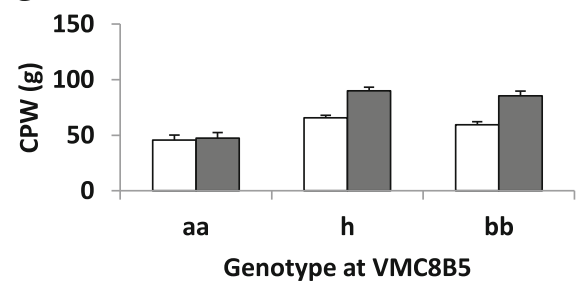

b

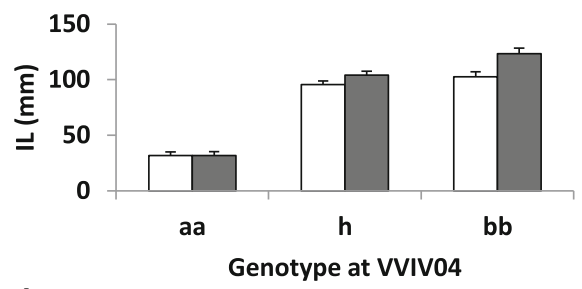

d

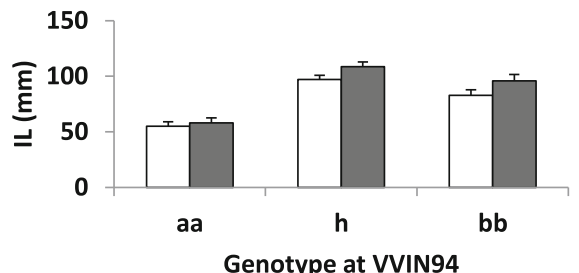

f

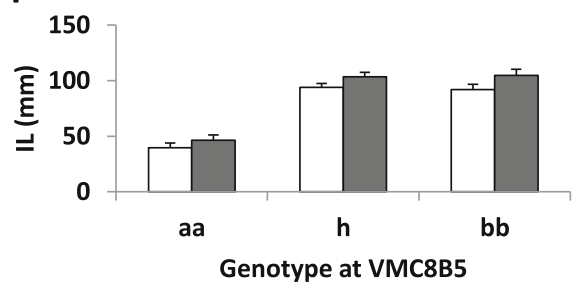

Fig. 4 Relationships between the genotypes at the WIV04, WIN94 and VMC8B5 loci and the cane pruning weight (CPW) and internode length (IL) traits. The CPW (a, c, e) and the IL (b, d, f) were expressed in $\mathrm{g}$ and in $\mathrm{mm}$ respectively (white bars 2009, grey bars 2010). Vertical bars indicate standard errors. aa, homozygous with CS allele; h, heterozygous; bb, homozygous with RGM allele

Some of the most strongly up-regulated transcripts were MADS box transcription factors: VIT_210s0003g02070, the grapevine orthologue of $A$. thaliana AGAMOUS SHATTERPROOF1 (VviAG SHP1), VIT_214s0083g01050 the grapevine orthologue of $A$. thaliana SEPALLATA1 (VviSEP1), VIT 201s0010g03900 the grapevine orthologue of AtSEP3, VIT $212 s 0142 g 00360$ the grapevine orthologue of $A$. thaliana SHATTERPROOF1 (VviSHP1) and VIT_217s0000g05000 (VviSEP2). A further 7 transcription factors were upregulated in the dwarfed plants including one MYB transcription factor (VIT_217s0000g09080, VviMYB50/Vvi$M Y B 55 /$ viMYB86), three zinc finger homeobox domain transcription factors, MINI ZINC FINGER1 (VIT 217s0000g06200, VviMIF1), VviMIF2B (VIT_214s0108g00 810) and VviMIF2A (VIT_214s0108g00760), and one BEL1related homeobox transcription factor (VIT_206s0004g02 580, VviBLH8). VviMIF2A and VviMIF2B genes are located on chromosome 14 within the \pm 2 -LOD confidence interval of the QTL for IL. The only transcript differentially expressed between the dwarf and normal plants within the \pm 2 -LOD confidence interval of the QTL for CPW and IL on chromosome 18 was a beta-caryophyllene synthase (VIT 218s0001g04830, TPS21/terpene synthase 21).

Four of the most strongly down-regulated transcripts are from genes which are contiguously located on chromosome 7 , within the confidence interval of the
QTLs for IL and CPW. Low hybridization signals, not different of background noise, were detected for these four genes demonstrating an absence of expression. These four genes are a SET domain-containing protein that is the orthologue of A. thaliana CURLY LEAF (VIT_207s0031g00320, VviCLF), a gene belonging to the phospholipase C-like phosphodiesterases superfamily (VIT_207s0031g00330), an F-box family protein (VIT_207s0031g00340) and a caffeoyl-CoA Omethyltransferase (VIT_207s0031g00350, VviCCoAOMT). This result combined with the 'aa' allelic form of VMC1A12 and VVIV04 suggested the presence of a deletion in CS genome.

\section{An $\mathbf{8 4 , 4 8 2}$ bp deletion was identified on chromosome 7 of dwarfed genotypes}

The BAC end sequences of eight CS BAC clones were used to select in silico clones surrounding the confidence interval of the QTLs for IL and CPW on LG7. After digestion, an estimation of the insert size of each BAC was done. Two BAC clones, VVCS1H006A20 and VVCS1H018A11, without and with deletion, were selected for a complete PacBio sequencing. According to in silico analyses of BAC end sequence positions, the insert size of BAC clone VVCS1H006A20 was evaluated as $140 \mathrm{~kb}$, the expected size. However, the insert size of the VVCS1H018A11 clone should have a length of $236.1 \mathrm{~kb}$, 
Table 2 Transcripts differentially expressed in the shoot tips between normal and dwarfed individuals of the Vitis vinifera cv. Cabernet-Sauvignon x V. riparia cv. Gloire de Montpellier F2 population (CS x RGM_F2)

\begin{tabular}{|c|c|c|c|c|c|c|c|}
\hline \multirow[b]{2}{*}{ Microarray identifier } & \multicolumn{3}{|c|}{ Expression level $(\log 2)$} & \multirow[b]{2}{*}{$\begin{array}{l}p \text { value } \\
\text { adjusted } \\
\text { with Holm }\end{array}$} & \multirow[b]{2}{*}{ Gene identifier V2 } & \multirow[b]{2}{*}{ Gene name } & \multirow[b]{2}{*}{ CRIBI annotation } \\
\hline & $\begin{array}{l}\text { Mean } \\
\text { Dwarfed }\end{array}$ & $\begin{array}{l}\text { Mean } \\
\text { Normal }\end{array}$ & $\begin{array}{l}\text { Dwarf - } \\
\text { Normal }\end{array}$ & & & & \\
\hline $\begin{array}{l}\text { CHRUN_JGW1308_ } \\
\text { 2_T01 }\end{array}$ & 5.2 & 11.1 & -6.0 & 0.00 & $\begin{array}{l}V_{1 T} \\
200 \text { s1308g00020 }\end{array}$ & & upf0308 protein chloroplastic-like \\
\hline $\begin{array}{l}\text { CHRUN_JGW566_1_ } \\
\text { T01 }\end{array}$ & 5.0 & 10.4 & -5.4 & 0.00 & $\begin{array}{l}\text { VIT_} \\
200 s 0566 \mathrm{~g} 00010\end{array}$ & & upf0308 protein chloroplastic-like \\
\hline $\begin{array}{l}\text { CHR7_JGW31_29_ } \\
\text { T01 }\end{array}$ & 5.3 & 10.6 & -5.4 & 0.00 & $\begin{array}{l}\text { VIT- } \\
207 \text { s0031g00320 }\end{array}$ & CLF/CURLY LEAF & $\begin{array}{l}\text { polycomb protein ez } 1 \text { set domain } \\
\text { protein }\end{array}$ \\
\hline $\begin{array}{l}\text { CHR7_JGW31_32_ } \\
\text { T01 }\end{array}$ & 6.2 & 11.3 & -5.1 & 0.00 & $\begin{array}{l}\text { VIT_ } \\
207 s 0031 \mathrm{~g} 00350\end{array}$ & CCOAOMT & caffeoyl-coa o-methyltransferase \\
\hline $\begin{array}{l}\text { CHR7_JGW31_30_ } \\
\text { T01 }\end{array}$ & 6.2 & 10.7 & -4.5 & 0.00 & $\begin{array}{l}\text { VIT_} \\
207 s 0031 g 00330\end{array}$ & & $\begin{array}{l}\text { pi-plc } x \text { domain-containing protein } \\
\text { at5g67130-like }\end{array}$ \\
\hline $\begin{array}{l}\text { CHR7_JGW31_31_ } \\
\text { T01 }\end{array}$ & 5.7 & 9.5 & -3.8 & 0.00 & $\begin{array}{l}\text { VIT_ } \\
207 \text { s0031g00340 }\end{array}$ & & f-box protein \\
\hline $\begin{array}{l}\text { CHR7_JGW5_241_ } \\
\text { T01 }\end{array}$ & 5.9 & 8.9 & -3.0 & 0.03 & $\begin{array}{l}\text { VIT_ } \\
207 s 0005 \mathrm{~g} 02490\end{array}$ & CYP709B2 & cytochrome p450 \\
\hline $\begin{array}{l}\text { CHR5_JGW77_76_ } \\
\text { T01 }\end{array}$ & 5.6 & 7.5 & -1.9 & 0.03 & $\begin{array}{l}\text { VIT_ } \\
205 \text { s0077g01020 }\end{array}$ & & $\begin{array}{l}\text { probable } n \text {-acetyltransferase hook- } \\
\text { less } 1\end{array}$ \\
\hline $\begin{array}{l}\text { CHR7_JGW129_32_ } \\
\text { T01 }\end{array}$ & 11.3 & 10.3 & 1.0 & 0.05 & $\begin{array}{l}\text { VIT_ } \\
207 \text { s0129g00290 }\end{array}$ & & Formamidase \\
\hline $\begin{array}{l}\text { CHR17_JGWO_115_ } \\
\text { T01 }\end{array}$ & 9.0 & 7.8 & 1.1 & 0.01 & $\begin{array}{l}\text { VIT- } \\
217 s 0000 g 09080\end{array}$ & MYB50 MYB55 MYB86 & $\begin{array}{l}\text { hypothetical protein r2r3-myb } \\
\text { transcription }\end{array}$ \\
\hline $\begin{array}{l}\text { CHR2_JGW87_15_ } \\
\text { T01 }\end{array}$ & 12.3 & 11.1 & 1.2 & 0.05 & $\begin{array}{l}\text { VIT } \\
202 s 0087 g 00840\end{array}$ & ABCG14 & $\begin{array}{l}\text { white-brown-complex abc } \\
\text { transporter family }\end{array}$ \\
\hline $\begin{array}{l}\text { CHR8_JGW7_740_ } \\
\text { T01 }\end{array}$ & 11.1 & 9.9 & 1.2 & 0.03 & $\begin{array}{l}\text { VIT_ } \\
\text { 208s0007g01180 }\end{array}$ & & receptor protein kinase 1-like \\
\hline $\begin{array}{l}\text { CHR8_JGW40_125_ } \\
\text { T01 }\end{array}$ & 6.2 & 5.0 & 1.2 & 0.02 & $\begin{array}{l}\text { VIT_ } \\
208 \mathrm{~s} 0040 \mathrm{~g} 02020\end{array}$ & FLA11 & $\begin{array}{l}\text { fasciclin-like arabinogalactan } \\
\text { protein 11-like }\end{array}$ \\
\hline $\begin{array}{l}\text { CHR13_JGW19_208_ } \\
\text { T01 }\end{array}$ & 11.6 & 10.0 & 1.5 & 0.02 & $\begin{array}{l}\text { VIT_ } \\
213 \text { s0019g03130 } \\
\text { VIT_- } \\
213 \mathrm{~s} 0019 \mathrm{~g} 03120\end{array}$ & UGT85A2/ UGT85A1 & udp-glycosyltransferase $85 a 1$ \\
\hline $\begin{array}{l}\text { CHR13_JGW106_5_ } \\
\text { T01 }\end{array}$ & 9.9 & 8.3 & 1.5 & 0.03 & $\begin{array}{l}\text { VIT__ } \\
213 s 0106 g 00060\end{array}$ & & ankyrin repeat-containing \\
\hline CHR6_JGW9_33_T01 & 12.7 & 11.1 & 1.6 & 0.04 & $\begin{array}{l}\text { VIT__ } \\
206 s 0009 g 03450\end{array}$ & LPR1 & lateral root primordium protein \\
\hline $\begin{array}{l}\text { CHR16_JGW22_167_ } \\
\text { T01 }\end{array}$ & 6.6 & 5.0 & 1.6 & 0.01 & $\begin{array}{l}\text { VIT_- } \\
216 s 0022 g 00560\end{array}$ & & $\begin{array}{l}\text { paired amphipathic helix protein } \\
\sin 3\end{array}$ \\
\hline $\begin{array}{l}\text { CHR7_- } \\
\text { GSVIVT00000186001_- } \\
\text { T01 }\end{array}$ & 9.1 & 7.5 & 1.6 & 0.03 & $\begin{array}{l}\text { VIT_ } \\
207 \mathrm{~s} 0129 \mathrm{~g} 00750\end{array}$ & & isoflavone 2 -hydroxylase \\
\hline $\begin{array}{l}\text { CHR19_JGW85_28_ } \\
\text { T01 }\end{array}$ & 9.0 & 7.3 & 1.8 & 0.04 & $\begin{array}{l}\text { VIT_ } \\
219 s 0085 \mathrm{~g} 00950\end{array}$ & NAC028 & nac domain ipr003441 \\
\hline $\begin{array}{l}\text { CHR3_JGW17_56_ } \\
\text { T01 }\end{array}$ & 8.8 & 7.0 & 1.8 & 0.00 & $\begin{array}{l}\text { VIT_- } \\
203 s 0017 g 01010\end{array}$ & AMC1/Metacaspase-1 & hypothetical protein \\
\hline $\begin{array}{l}\text { CHR18_RANDOM_ } \\
\text { JGW82_56_T01 }\end{array}$ & 8.2 & 6.3 & 1.9 & 0.01 & $\begin{array}{l}\text { VIT_ } \\
218 \mathrm{~s} 0001 \mathrm{~g} 04830\end{array}$ & $\begin{array}{l}\text { TPS21/terpene synthase } \\
21\end{array}$ & beta-caryophyllene synthase \\
\hline $\begin{array}{l}\text { CHR4_JGW43_22_ } \\
\text { T01 }\end{array}$ & 7.1 & 5.0 & 2.1 & 0.02 & $\begin{array}{l}\text { VIT_- } \\
204 s 0043 g 00300\end{array}$ & & $\begin{array}{l}\text { hypothetical protein } \mathrm{tp} \times 2 \\
\text { (targeting protein for } \times k \mid \mathrm{l} 2 \text { ) family } \\
\text { protein }\end{array}$ \\
\hline $\begin{array}{l}\text { CHR1_JGW150_30_ } \\
\text { T01 }\end{array}$ & 8.1 & 6.1 & 2.1 & 0.05 & $\begin{array}{l}\text { VIT_ } \\
20150150 \mathrm{~g} 00300\end{array}$ & $\mathrm{GH} 3$ & $\begin{array}{l}\text { indole-3-acetic acid-amido } \\
\text { synthetase }\end{array}$ \\
\hline CHR4_ & 7.9 & 5.7 & 2.2 & 0.05 & VIT_ & & hypothetical protein \\
\hline
\end{tabular}


Table 2 Transcripts differentially expressed in the shoot tips between normal and dwarfed individuals of the Vitis vinifera cv. Cabernet-Sauvignon x V. riparia cv. Gloire de Montpellier F2 population (CS x RGM_F2) (Continued)

\begin{tabular}{|c|c|c|c|c|c|c|c|}
\hline \multirow[b]{2}{*}{ Microarray identifier } & \multicolumn{3}{|c|}{ Expression level (log2) } & \multirow[b]{2}{*}{$\begin{array}{l}p \text { value } \\
\text { adjusted } \\
\text { with Holm }\end{array}$} & \multirow[b]{2}{*}{ Gene identifier V2 } & \multirow[b]{2}{*}{ Gene name } & \multirow[b]{2}{*}{ CRIBI annotation } \\
\hline & $\begin{array}{l}\text { Mean } \\
\text { Dwarfed }\end{array}$ & $\begin{array}{l}\text { Mean } \\
\text { Normal }\end{array}$ & $\begin{array}{l}\text { Dwarf - } \\
\text { Normal }\end{array}$ & & & & \\
\hline $\begin{array}{l}\text { GSVIVT00035889001_ } \\
\text { T01 }\end{array}$ & & & & & $204 s 0008 g 05790$ & & \\
\hline $\begin{array}{l}\text { CHR7_JGW129_29_ } \\
\text { T01 }\end{array}$ & 10.6 & 8.4 & 2.2 & 0.02 & $\begin{array}{l}\text { VIT-_ } \\
207 s 0129 \mathrm{~g} 00290\end{array}$ & & formamidase \\
\hline $\begin{array}{l}\text { CHRUN_ } \\
\text { GSVIVT00006381001_ } \\
\text { T01 }\end{array}$ & 8.7 & 6.5 & 2.3 & 0.04 & $\begin{array}{l}\text { VIT_- } \\
200 \text { s0358g00020 }\end{array}$ & & hypothetical protein \\
\hline $\begin{array}{l}\text { CHR13_RANDOM_ } \\
\text { JGW112_22_T01 }\end{array}$ & 11.2 & 8.9 & 2.3 & 0.01 & $\begin{array}{l}\text { VIT } \\
213 \text { s0067g00620 }\end{array}$ & & $\begin{array}{l}\text { bifunctional dihydroflavonol 4- } \\
\text { reductase flavanone 4-reductase }\end{array}$ \\
\hline $\begin{array}{l}\text { CHR8_JGW40_238_ } \\
\text { T01 }\end{array}$ & 11.8 & 9.4 & 2.5 & 0.04 & $\begin{array}{l}\text { VIT } \\
208 s 0040 g 00820\end{array}$ & CYP94D2 & cytochrome p450 \\
\hline $\begin{array}{l}\text { CHR6_JGW4_547_ } \\
\text { T01 }\end{array}$ & 11.1 & 8.5 & 2.6 & 0.00 & $\begin{array}{l}\text { VIT_- } \\
206 s 0004 g 02580\end{array}$ & BLH8 & $\begin{array}{l}\text { bel1 homeotic hypothetical } \\
\text { protein }\end{array}$ \\
\hline $\begin{array}{l}\text { CHR2_JGW25_23_- } \\
\text { T01 }\end{array}$ & 10.6 & 7.9 & 2.7 & 0.00 & $\begin{array}{l}\text { VIT_ } \\
202 s 0025 \mathrm{~g} 00250\end{array}$ & SP1L5/SPIRAL1-like 5 & nitrilase-associated protein \\
\hline $\begin{array}{l}\text { CHR16_PDW115_ } \\
\text { 38_T01 }\end{array}$ & 8.7 & 5.9 & 2.8 & 0.00 & $\begin{array}{l}\text { VIT } \\
21 \overline{6 s} 0115 \mathrm{~g} 00410\end{array}$ & & hypothetical protein \\
\hline $\begin{array}{l}\text { CHR14_- } \\
\text { GSVIVT00030939001_ } \\
\text { T01 }\end{array}$ & 8.6 & 5.8 & 2.8 & 0.00 & $\begin{array}{l}\text { VIT_ } \\
214 s 0006 \mathrm{~g} 02160\end{array}$ & & $\begin{array}{l}\text { probable s-adenosylmethionine- } \\
\text { dependent methyltransferase } \\
\text { at5g37990 }\end{array}$ \\
\hline $\begin{array}{l}\text { CHR8_JGW40_281_ } \\
\text { T01 }\end{array}$ & 10.4 & 7.5 & 2.9 & 0.02 & $\begin{array}{l}\text { VIT_ } \\
208 s 0040 \mathrm{~g} 00380\end{array}$ & & $\begin{array}{l}\text { probable s-acyltransferase } \\
\text { at5g05070-like }\end{array}$ \\
\hline $\begin{array}{l}\text { CHR15_JGW24_108_ } \\
\text { T01 }\end{array}$ & 9.4 & 6.5 & 2.9 & 0.00 & $\begin{array}{l}\text { VIT_} \\
215 \mathrm{~s} 0024 \mathrm{~g} 00440\end{array}$ & & disease resistance protein rga3-like \\
\hline $\begin{array}{l}\text { CHR14_JGW83_93_ } \\
\text { T01 }\end{array}$ & 8.5 & 5.5 & 3.0 & 0.00 & $\begin{array}{l}\text { VIT- } \\
214 s 0083 g 01050\end{array}$ & SEP1 & mads-box protein \\
\hline $\begin{array}{l}\text { CHR8_JGW7_766_ } \\
\text { T01 }\end{array}$ & 11.9 & 8.9 & 3.0 & 0.01 & $\begin{array}{l}\text { VIT_ } \\
208 s 0007 g 00890\end{array}$ & & $\begin{array}{l}\text { tropinone reductase homolog } \\
\text { at1g07440 }\end{array}$ \\
\hline $\begin{array}{l}\text { CHR7_JGW31_36_ } \\
\text { T01 }\end{array}$ & 8.4 & 5.3 & 3.0 & 0.02 & $\begin{array}{l}\text { VIT_ } \\
207 \text { s0031g00400 }\end{array}$ & & zinc finger protein \\
\hline $\begin{array}{l}\text { CHR12_JGW35_122_ } \\
\text { T01 }\end{array}$ & 8.3 & 5.1 & 3.3 & 0.01 & $\begin{array}{l}\text { VIT } \\
212 s 0035 g 00900\end{array}$ & $\begin{array}{l}\text { JAZ12/jasmonate-zim- } \\
\text { domain protein } 12\end{array}$ & protein tify $3 b$ \\
\hline $\begin{array}{l}\text { CHR5 } \\
\text { GSVIVT00029081001_- } \\
\text { T01 }\end{array}$ & 8.6 & 4.9 & 3.7 & 0.00 & $\begin{array}{l}\text { VIT_- } \\
205 \text { s0051g00880 }\end{array}$ & & hypothetical protein \\
\hline $\begin{array}{l}\text { CHRUN_ } \\
\text { GSVIVT00003451001_ } \\
\text { T01 }\end{array}$ & 8.2 & 4.4 & 3.8 & 0.00 & $\begin{array}{l}\text { VIT_ } \\
200 \text { s0193g00010 }\end{array}$ & & hypothetical protein \\
\hline $\begin{array}{l}\text { CHR19_JGW15_10_ } \\
\text { T01 }\end{array}$ & 9.2 & 5.3 & 3.8 & 0.00 & $\begin{array}{l}\text { VIT } \\
219 \mathrm{~s} 0015 \mathrm{~g} 00100\end{array}$ & CYP71B7 & cytochrome p450 \\
\hline $\begin{array}{l}\text { CHR17_JGW0_480_ } \\
\text { T01 }\end{array}$ & 9.1 & 5.2 & 3.9 & 0.00 & $\begin{array}{l}\text { VIT } \\
217 s 0000 g 05000\end{array}$ & SEP2 & sepallata1-like protein \\
\hline $\begin{array}{l}\text { CHR14_JGW108_72_ } \\
\text { T01 }\end{array}$ & 10.2 & 6.1 & 4.1 & 0.00 & $\begin{array}{l}\text { VIT_- } \\
214 s 0108 \mathrm{~g} 00760\end{array}$ & MIF2A & $\begin{array}{l}\text { zf-hd homeobox protein } \\
\text { at4g24660-like }\end{array}$ \\
\hline $\begin{array}{l}\text { CHR1_JGW11_153_ } \\
\text { T01 }\end{array}$ & 9.4 & 5.2 & 4.2 & 0.01 & $\begin{array}{l}\text { VIT_ } \\
201 s 0011 \mathrm{~g} 05100\end{array}$ & MLP34 & major latex \\
\hline $\begin{array}{l}\text { CHR10_JGW3_187_ } \\
\text { T01 }\end{array}$ & 13.7 & 9.4 & 4.3 & 0.00 & $\begin{array}{l}\text { VIT_ } \\
210 \mathrm{~s} 0003 \mathrm{~g} 02070\end{array}$ & AG SHP1 & agamous-like protein \\
\hline $\begin{array}{l}\text { CHRUN_JGW193_1_ } \\
\text { T01 }\end{array}$ & 9.9 & 5.6 & 4.3 & 0.00 & $\begin{array}{l}\text { VIT_- } \\
200 s 0193 g 00020\end{array}$ & & hypothetical protein \\
\hline CHR17_JGW0_372_ & 10.3 & 5.7 & 4.5 & 0.00 & VIT_ & MIF1 & zf-hd homeobox protein \\
\hline
\end{tabular}


Table 2 Transcripts differentially expressed in the shoot tips between normal and dwarfed individuals of the Vitis vinifera cv. Cabernet-Sauvignon x V. riparia cv. Gloire de Montpellier F2 population (CS x RGM_F2) (Continued)

\begin{tabular}{|c|c|c|c|c|c|c|c|}
\hline \multirow[b]{2}{*}{ Microarray identifier } & \multicolumn{3}{|c|}{ Expression level $(\log 2)$} & \multirow[b]{2}{*}{$\begin{array}{l}p \text { value } \\
\text { adjusted } \\
\text { with Holm }\end{array}$} & \multirow[b]{2}{*}{ Gene identifier V2 } & \multirow[b]{2}{*}{ Gene name } & \multirow[b]{2}{*}{ CRIBI annotation } \\
\hline & $\begin{array}{l}\text { Mean } \\
\text { Dwarfed }\end{array}$ & $\begin{array}{l}\text { Mean } \\
\text { Normal }\end{array}$ & $\begin{array}{l}\text { Dwarf- } \\
\text { Normal }\end{array}$ & & & & \\
\hline T01 & & & & & $217 \mathrm{~s} 0000 \mathrm{~g} 06200$ & & at4g24660-like \\
\hline $\begin{array}{l}\text { CHR15_JGW24_104_ } \\
\text { T01 }\end{array}$ & 10.0 & 5.0 & 4.9 & 0.00 & $\begin{array}{l}\text { VIT_ } \\
215 \mathrm{~s} 0024 \mathrm{~g} 00480\end{array}$ & PP2-A1 & protein phloem protein 2 -like a1 \\
\hline $\begin{array}{l}\text { CHR12_JGW142_18_ } \\
\text { T01 }\end{array}$ & 12.5 & 7.0 & 5.5 & 0.00 & $\begin{array}{l}V_{1 T} \\
212 \mathrm{~s} 0142 \mathrm{~g} 00360\end{array}$ & SHP1 & agamous-like protein \\
\hline $\begin{array}{l}\text { CHR14_JGW81_14_ } \\
\text { T01 }\end{array}$ & 11.9 & 6.2 & 5.7 & 0.00 & $\begin{array}{l}\text { VIT- } \\
214 s 0081 g 00670\end{array}$ & $\begin{array}{l}\text { AHL19, AT-hook motif } \\
\text { nuclear-localized pro- } \\
\text { tein } 19\end{array}$ & dna-binding protein escarola-like \\
\hline $\begin{array}{l}\text { CHR14_JGW108_75_ } \\
\text { T01 }\end{array}$ & 12.0 & 6.0 & 6.0 & 0.00 & $\begin{array}{l}V_{1 T} \\
214 s 0108 g 00810\end{array}$ & MIF2B & $\begin{array}{l}\text { zf-hd homeobox protein } \\
\text { at4g24660-like }\end{array}$ \\
\hline $\begin{array}{l}\text { CHR1_JGW10_295_ } \\
\text { T01 }\end{array}$ & 13.1 & 5.7 & 7.4 & 0.00 & $\begin{array}{l}\text { VIT_ } \\
20150010 g 03900\end{array}$ & SEP3 & transcription factor \\
\hline
\end{tabular}

Log2 fold change $>1, p$ value $<0.05$ adjusted with Holm, $n=5$

but the digestion of this clone revealed an insert size of only $145 \mathrm{~kb}$, suggesting a deletion of approximately 91.1 $\mathrm{kb}$.

After PacBio sequencing, the length of the insert sequences of the BAC clones VVCS1H0O6A2O and VVCS1H018A11 was $138,300 \mathrm{bp}$ and 145,215 bp respectively. These sequences were compared to $12 X . v 2$ genome sequence (Additional files 6 and 7). The insert sequence of VVCS1H006A20 aligned from 22,790,285 to 22,944,987 bp of the chromosome 7 without large deletions and genome rearrangements. The insert sequence of VVCS1H018A11 aligned from $22,749,398$ to $24,724,688 \mathrm{bp}$ of the chromosome 7 sequence. Two large deletions of 13,084 bp and 84, 482 bp were shown (Additional file 8). The first deletion was located between 22,843,538 and 22,856,622 bp on the chromosome 7 . Within this chromosomal region, only one potentially expressed gene without known function, VIT 207s0031g00285, was located. No probe of this gene was present on the microarray. The second deletion was observed between 22,863,166 and 22,947,648 bp. Within this chromosomal region, 10 potentially expressed genes were located and among them the four not expressed genes previously described (Additional file 8). The expression of the other 6 potentially expressed genes was not detected in either the normal or dwarfed plants in the tissue studied as for VIT_207s0031g00285 (data not shown). This deletion was replaced by an insertion of a $5587 \mathrm{bp}$-long sequence which was at $26 \%$ composed with (TTA)n simple repeats of satellite DNA. We discovered two transpositions of about $600 \mathrm{bp}$ within the region of the first deletion. The related sequences were composed of AT-rich satellite DNA and located between 22,868,133 and 22,868,768 bp and between $24,724,075$ and $24,724,688$ bp on the chromosome 7 of the 12X.v2 grape genome. The first transposition was also associated with an inversion (Additional files 7 and 8). Based on
RepeatMasker2.1 results, partial direct repeats were present near the deletion junction regions.

Presence of the $\mathbf{8 4 , 4 8 2}$ bp deletion in grapevine cultivars The presence of the deletion of $84,482 \mathrm{bp}$ on chromosome 7 was confirmed in the dwarfed individuals by PCR analysis using primers that flank the predicted deletion site in CS BAC clone VVCS1H018A11. As expected, DNAs from BAC clone VVCS1H018A11, dwarfed genotypes or CS cultivar produced a $782 \mathrm{bp}$ PCR product, while DNA from normal individuals with a homozygous 'bb' genotype at the VVIV04 locus failed to produce any PCR products because the DNA fragment between both primers was too long to be amplified.

The presence of the deletion named 'Delchr7' within the genome of 51 Vitis vinifera cultivars was investigated thanks to the PCR-based marker (Additional file 9). The deletion was found in heterozygous form in the genome of only three cultivars: Sauvignon, CS and Arinarnoa.

\section{Discussion}

Three loci explain $62.5 \%$ of the total variance in IL

Using an interspecific $V$. vinifera $x V$. riparia F2 population with $17 \%$ of dwarfed and abnormal individuals, we identified three loci related to IL on LG7, 14 and 18, explaining 33.0, 14.8 and $12.2 \%$ of the phenotypic variance, respectively. The transcriptomic analysis suggested that four genes within the interval of the QTL on LG7 were absent in the genome of dwarfed plants, which has been confirmed by BAC clone sequencing.

To the best of our knowledge, the only well characterized grapevine dwarf is the gibberellin insensitive $V v i$ gail mutation identified in $V$. vinifera $\mathrm{cv}$. Pinot meunier [3], which does not co-locate with any of our QTLs. In other species, studies of the genetic architecture of dwarfism and IL suggest that these traits are mainly under the control of genes belonging to two functional 
categories. Firstly and most frequently observed, genes coding for proteins are involved in hormone metabolism. For example, the $c p$ locus that confers a dwarf phenotype to cucumber and was shown by fine mapping to co-locate with a cytokinin oxydase gene [21]; the "Rinrei" mutant of faba bean, impaired on brassinosteroid biosynthetic gene $b d d 1$, which codes for a C-24 sterol reductase [22]; and the GmDW1 locus of soybean, which corresponds to an ent-kaurene synthase, one of the early steps of the gibberellin biosynthetic pathway [23]. Secondly, genes coding transcription factors involved in the regulation of shoot development and architecture, such as Reduced height in wheat, that codes for a DELLA transcription factor [24]; or the dil1 locus in maize that was shown by map-based cloning to correspond to AP2like gene [25]. None of the above-mentioned genes were found in the confidence intervals of our QTLs.

\section{Four genes located inside the $\mathbf{8 4 , 4 8 2}$ bp deletion of} chromosome 7 were not expressed in the dwarfed plants Among 11 genes deleted on chromosome 7 of the dwarfed plants, nine are present on the microarray and two were quantified by qPCR. Of these 11 genes, the transcripts of four genes were not expressed in the plants with a dwarfed phenotype, but were expressed in normal individuals; they were VviCLF, VIT_207s0031g00330,VIT_207s0031g00340 and VviCCoAOMT. CLF is a well-described developmental regulator that participates in transcriptional repression via methylation of histone $\mathrm{H} 3$ lysine 27 (H3K27) in the polycomb repressive complex 2 in A. thaliana [26]. Mutants of CLF show early flowering and curled leaves, and this phenotype is largely caused by the mis-expression of the floral homeotic gene $A G$ in leaves [27]. In wild type plants, $A G$ is expressed only in flowers where it specifies the identity of stamens and carpels. The absence of VviCLF in the dwarfed grapevine plants studied here was associated with plants with curled, small leaves and the up-regulation of VviAG SHP1 expression in vegetative shoot tissue. In addition to the mis-expression of VviAG $S H P 1$, a number of other floral homeotic genes were upregulated in the dwarfed plants such as VviSEP1, VviSEP2, VviSEP3 and VviSHP1. The mis-expression of various flower identity and flowering time control genes have also been reported in $A$. thaliana clf and ag mutants, such as the mis-expression of AtAP2, AtSHP1, AtSHP2, AtSEP3, FLOWERING LOCUS T and FLOWERING LOCUS C [26-28]. This could suggest that VviCLF protein has similar functions in grapevine to that of AtCLF in terms of floral gene repression in vegetative tissues in A. thaliana.

Reproductive development in grapevine differs significantly from that of annual plants such as A. thaliana. In temperate regions, floral initiation occurs in the spring/ summer in latent buds and these buds remain dormant over the following winter. Mature flowers develop from immature primordia at bud break. $V v i C L F$ is highly expressed in latent bud during the flower initiation and at bud break, and is not expressed during the dormant period [29]. Like its $A$. thaliana orthologue, VviCLF is also highly expressed in vegetative tissue such as leaves and tendrils [29]. Potentially the mis-expression of floral homeotic genes in the dwarfed plants could be responsible for the absence of flowers in these plants. VIT_207s0031g00330 was absent from the dwarfed plants, VIT_207s0031g00330 is a phospholipase C-like phosphodiesterases superfamily protein. Phospholipase C-like phosphodiesterases are intracellular enzymes with important roles in signal transduction processes [30], but the function of most proteins remains unknown. VIT_207s0031g00340 is an F-box protein; these proteins generally provide substrate specificity for Skp1-Cullin-F-Box complexes that direct protein degradation via the ubiquitin$26 \mathrm{~S}$ proteasome pathway [31]. F-box proteins are responsible for the regulation of a wide range of biological processes and there are $156 \mathrm{~F}$-box proteins present in the grapevine genome [32], as such, it is difficult to assign a putative function to VIT_207s0031g00340. Caffeoyl-CoA O-methyltransferases have essential roles in lignin biosynthesis in both herbaceous and woody plants [33]. A. thaliana mutants in CCoAOMT1 shows slightly reduced development under short-day conditions, but no visual phenotype under long days [34]. Poplar trees with reduced CCoAOMT activity exhibit no obvious visible phenotype, yet reduced lignin contents [35]. The loss of $V v i C C O A O M T$ in the dwarfed plants was associated with the up-regulation of expression of an arabinogalactan protein (VTT_208s0040g02020) and a cinnamyl alcohol dehydrogenase (VIT_213s0067g00620), a similar result has been observed in ccoaomt1 mutants of $A$. thaliana (an arabinogalactan protein and a cinnamoyl-CoA reductase were upregulated) [36]. This may suggest that the loss of a CCoAOMT triggers some degree of cell wall modification in the dwarfed plants which is similar to that of $A$. thaliana ccoaomt mutants.

\section{VviMIF2A and VviMIF2B are up-regulated in the dwarfed plants and are located within the QTL of IL on LG 14} In addition to the differential accumulation of transcripts of the floral regulators cited above, a number of transcription factors were differentially expressed between the dwarfed and normal individuals, including the up-regulation of three zinc finger homeobox transcription factors VviMIF1, VviMIF2A and VviMIF2B. MIF1 is known to regulate plant hormone signalling pathways and MIF1 over-expressing A. thaliana plants (35S:: MIF1) show dwarf phenotypes with reduced apical dominance, dark-green leaves, curled leaves, altered flower morphology, poor fertility and spoon like cotyledons [37]. 35S::MIF2 and 35S::MIF3 lines have similar visible phenotypes to those of 35S::MIF1 [38]. It was also suggested that MIF1 and MIF3 have roles in meristem 
formation as severe $35 S:: M I F 1$ or $35 S::$ MIF3 plants have ectopic shoot meristems on leaf margins and develop ovules along the edges of sepals [38]. The phenotype of MIF over-expressers is similar to that of the dwarfed grapevines in this study suggesting that the increase in MIF transcription factors could also explain some of the phenotypic characteristics of the dwarfed plants. Furthermore, VviMIF $2 A$ and $V v i$ $M I F 2 B$ are within the confidence interval of the QTL for IL on LG14 suggesting that they may directly control some part of the dwarfed phenotype.

\section{Genetic origin of the 84,482 bp deletion on chromosome 7 of dwarfed plants}

The 84,482 bp deletion on chromosome 7 was unique to the CS genome and other insertions/deletions were not found in the RGM genome at Delchr7 locus which was homozygous [39]. Considering that the recessive locus was given by the female $V$. vinifera parent of the F2 progeny, the presence of the 84,482 bp deletion was investigated in the genome of 51

$V$. vinifera varieties. The deletion 'Delchr7' was found in the genome of three of the 11 members of the kin group of Savagnin: Sauvignon, CS (which is a progeny of Sauvignon and the mother of the F2 progeny studied here), and Arinarnoa (which is a progeny of CS). Taking into account the parentage of the investigated cultivars [40-42], this shows that 'Delchr7' of CS came from the unknown parent of Sauvignon or was the result of meiotic events during the genetic cross between Savagnin and this unknown parent (Fig. 5). Savagnin has probably crossed with a single, unknown and probably extinct variety to give birth to the siblings Sauvignon,
Trousseau and Chenin [41]. However, only Sauvignon received 'Delchr7' in its genome and transmitted it to its offspring. The 'Delchr7' locus does not appear to have a negative impact on shoot development when this locus is heterozygous (i.e. in Sauvignon, CS and Arinarnoa), thus it may be concluded that 'Delchr7' is a deleterious recessive locus.

In addition to the 84,482 bp deletion, another large deletion of $13,084 \mathrm{bp}$, a transposition and a transversion were identified on chromosome 7 of dwarfed plants in comparison to the 12X.v2 genomic sequence. It is known that active mobile elements can cause chromosomal rearrangements, including genomic deletion [43]. Although partial direct repeats were identified near the deletion junction regions by in silico analysis, it is not clear whether they were involved in the formation of deletions.

Repetitive DNA sequences with a variable AT-rich repeat unit were identified within the inserted sequence replacing the 84,482 bp deletion region and within the two transposition sequences. Satellite DNAs are accumulated in the heterochromatin, mainly in centromeric and subtelomeric regions. Repetitive DNA sequences have developmental, cellular, and cytoplasmic effects and play a role in chromosomal recombination [44]. They are involved in several changes, such as transposition, segmental duplications and mechanisms based on rolling-circle replication of extrachromosomal circular DNAs and reinsertion [45]. Thus, the chromosomal rearrangements evidenced on chromosome 7 of CS $x$ RGM_F2 dwarfed plants could be a consequence of the presence of repetitive DNA sequences.

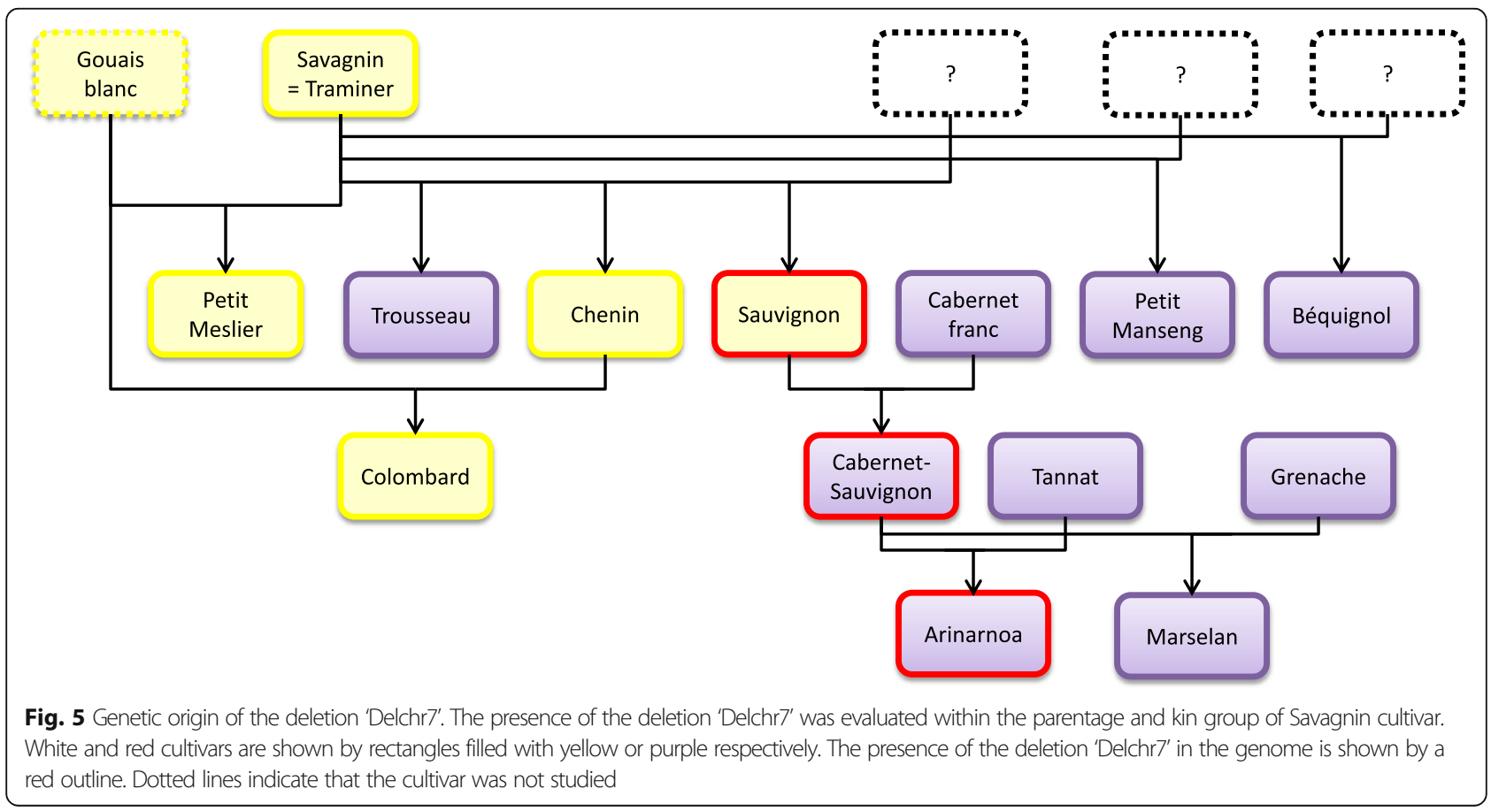




\section{Conclusions}

The combination of genetic and transcriptomic analyses facilitated the identification of a major deletion on chromosome 7 of CS which, when homozygous, appears largely responsible for dwarfing in an interspecific $V$. vinifera $\mathrm{x} V$. riparia F2 population. Other QTLs on LGs 14 and 18 were also identified for IL trait. The large deletion of $84,482 \mathrm{bp}$ on chromosome 7 of CS encompasses 10 genes, among them $V v i C L F$, a major developmental regulator that putatively suppresses the expression of floral homeotic genes in vegetative tissues. The deletion was found in relatives of CS such as Sauvignon, but is not widely distributed in grapevine cultivars. This work is the first molecular characterization of a deleterious recessive locus in grapevine potentially explaining dwarfed phenotypes in backcrosses or F2 populations with CS and its relatives. The PCR-based molecular marker 'DelChr7' defined in this study could now be used to track for the presence of the deletion in Sauvignon and CS based progenies, in order to limit the risk of appearance of abnormalities in subsequent crosses.

\section{Methods}

\section{Plant material}

The F2 population used in this study, named CS $\mathrm{x}$ RGM_F2, consisted of 337 individuals resulting from the inter-specific cross of $V$. vinifera $\mathrm{cv}$. CS $\mathrm{x} V$. riparia cv. RGM. This F2 population, developed in 2004 at INRA Bordeaux, France, derived from the self-fertilization of the F1_148 individual of the F1 CS X RGM1995-1 population [9]; itself obtained in 1995 at INRA Bordeaux, France, by a cross between $V$. vinifera $\mathrm{cv}$. CS and $V$. riparia cv. RGM [9].

The F1_148 individual and the CS x RGM_F2 population were maintained in pots in a naturally illuminated and semi-regulated greenhouse, with one plant per genotype. $V$. vinifera cultivars and $V$. riparia cv. RGM were present in vineyards at INRA Bordeaux, France. For all genotypes used in this study, leaves were collected in greenhouse and in vineyards according to institutional guidelines and directly used for total nucleic acid extraction. The identification of the $V$. vinifera cultivars and $V$. riparia $\mathrm{cv}$. RGM was done by the Institut Français de la Vigne et du Vin, France, by SSR markers. No permissions were required to obtain this plant material. SSR markers were also used at INRA Bordeaux, France, for the identification of the F1_148 individual [9] and the CS x RGM_F2 population.

\section{Total nucleic acid extraction}

Leaf samples (approximately $0.3 \mathrm{~g}$ fresh weight) were ground with a rolling grinder (HOMEX, Bioreba) in 5 $\mathrm{mL}$ of metabisulfite buffer containing $0.2 \mathrm{M}$ Tris- $\mathrm{HCl}$ pH 8.0, 70 mM EDTA pH 8.0, $2 \mathrm{M} \mathrm{NaCl}$ and $20 \mathrm{mM}$ sodium metabisulfite. Aliquots of leaf extracts $(0.5 \mathrm{~mL})$ were placed in tubes and $450 \mu \mathrm{L}$ of HATMAB buffer (2\% HATMAB w/v, 1.4 M NaCl, 20 mM EDTA pH 8.0, $100 \mathrm{mM}$ Tris- $\mathrm{HCl} \mathrm{pH} 8.0)$ was added. Tubes were vortexed vigorously, incubated for $1 \mathrm{~h}$ at $65^{\circ} \mathrm{C}$ and centrifuged at $1600 \mathrm{~g}$ for $25 \mathrm{~min}$ at $4{ }^{\circ} \mathrm{C}$. Five hundred $\mu \mathrm{L}$ of the supernatants were recovered and transferred to new tubes, followed by the addition of $450 \mu \mathrm{L}$ chloroformoctanol (24:1). The mixture obtained was vortexed and then incubated on ice with intermittent shaking. The tubes were centrifuged at $1600 \mathrm{~g}$ for $20 \mathrm{~min}$ at $4{ }^{\circ} \mathrm{C}$. Three hundred $\mu \mathrm{L}$ of the supernatant were recovered, and added with $150 \mu \mathrm{L} 10 \mathrm{M}$ ammonium acetate and $300 \mu \mathrm{L}$ isopropanol. Tubes were transferred at $-20{ }^{\circ} \mathrm{C}$ for $25 \mathrm{~min}$. Total nucleic acids were pelleted by centrifugation at $1600 \mathrm{~g}$ for $25 \mathrm{~min}$ at $4{ }^{\circ} \mathrm{C}$. Supernatants were removed and nucleic acid pellets were washed with ice cold $70 \% \mathrm{EtOH}$, air dried, and dissolved in $200 \mu \mathrm{L} 0.1 \mathrm{X}$ TE buffer.

\section{Choice of molecular markers}

SSR markers were mainly chosen from the markers used to construct the map of the F1 CS X RGM1995-1 population [9]. In addition, 46 new SSR markers were designed using the grape genome 12X sequence (http://www.genoscope.cns. fr/externe/GenomeBrowser/Vitis/, Additional file 1). Primers were designed with PRIMER0.5 software (Whitehead Institute for Biomedical Research). A new insertion-deletion marker VvOMT2-2 was also designed based on the VviOMT2 gene sequence [46]. A pair of primers, VvOMT2 2F:5' -AACTTTGCAGATGATAATCGAGG-3' and VvOM T2_2R:5'-ATGGATTCGACATTGAGAAAATG-3', were used to detect the presence an insertion-deletion of $7 \mathrm{bp}$ in the 3'UTR of VviOMT2 gene (VIT_12s0059g01750).

\section{Amplification of SSR molecular makers}

All PCR reactions were performed in $15 \mu \mathrm{L}$ reaction volume containing: $10 \mathrm{ng}$ of template DNA, 1x PCR reaction buffer, $2 \mathrm{mM} \mathrm{MgCl} 2,0.2 \mathrm{mM}$ of each dNTP, $0.2 \mu \mathrm{M}$ dye conjugated M13 primer, $0.05 \mu \mathrm{M}$ M13 tailed SSR forward primer, $0.2 \mu \mathrm{M}$ SSR reverse primer and $0.025 \mathrm{U}$ JumpStart $^{\mathrm{m}}$ Taq DNA Polymerase (Sigma). All PCR forward primer oligonucleotides were tailed on their 5'end with one of the following M13 forward sequences: A13, CACGACGTTGTAGGACCAC, B13, CACGTTCTGG AACATCGAC or C13, CACGCACTTGACGAAGGAC. Fluorescent dye (PET, NED, VIC or 6-FAM) was incorporated in amplicons by including a $5^{\prime}$ dye-labelled M13 forward primer in the PCR. PET, NED and VIC fluorescent dyes were associated to $\mathrm{A} 13, \mathrm{~B} 13$ and $\mathrm{C} 13$ respectively and FAM fluorescent dye with the three M13 forward sequences.

The PCR thermocycler conditions were the same for all primers pairs and adapted from the literature [9]: 5 min initial denaturation step at $94{ }^{\circ} \mathrm{C}$, followed by 3 
cycles of $30 \mathrm{~s}$ denaturation at $94{ }^{\circ} \mathrm{C}, 1 \mathrm{~min} 30 \mathrm{~s}$ annealing at $55^{\circ} \mathrm{C}$ or $60^{\circ} \mathrm{C}$ and $1 \mathrm{~min}$ extension at $72^{\circ} \mathrm{C}$, followed by 35 cycles of $30 \mathrm{~s}$ denaturation at $94{ }^{\circ} \mathrm{C}, 30 \mathrm{~s}$ annealing at $55^{\circ} \mathrm{C}$ or $60^{\circ} \mathrm{C}$ and $1 \mathrm{~min}$ extension at $72^{\circ} \mathrm{C}$ then followed by $7 \mathrm{~min}$ final extension at $72^{\circ} \mathrm{C}$.

SSR markers were first tested for amplicon marker quality with DNA samples obtained from CS, RGM, F1_148 and five individuals of the F2 population. Marker allele size ranges and single loci in the expected amplicon size ranges were evaluated. Polymorphic markers were then run on the entire CS X RGM_F2 mapping population.

A Hamilton STARlet robot (HAMILTON Robotics) was used to deposit $4 \mu \mathrm{L}$ genomic DNA of the each genotype of the CS x RGM_F2 population in 384-well PCR plates. PCR amplifications were made by multiplexing in single PCR reaction 2 to 4 markers sharing the same dye conjugated M13 primer. In a PCR reaction, markers sharing the same dye conjugated M13 primer could be only discriminated by their PCR product length.

\section{Marker evaluation and genotyping}

PCR amplicons and GeneScan ${ }^{\mathrm{TM}} 600 \mathrm{LIZ}^{\circ}$ dye internal size standards (Life Technologies Corporation) were separated by capillary electrophoresis using ABI 3730 (Life Technologies Corporation). Markers were multiplexed by 12 or 13 per capillary channel by combining with Hamilton STARlet robot aliquots of 4-6 PCR reactions. Allele sizing was performed with ABI PRISM GENEMAPPER 4.0 software (Life Technologies Corporation) according to the manual instructions.

\section{Linkage analysis and mapping}

The map was constructed using the software CarthaGene [47] at a logarithm of the odds (LOD) value of 5.0 and at a maximal distance threshold of $35 \mathrm{cM}$. Validation of the map obtained was done using the software JoinMap 3.0 [48] using a Kosambi's mapping function. The marker order obtained was checked according to the consensus map of the F1 population CS x RGM1995-1 and to the 12X genome sequence. The linkage groups (LGs) were numbered LG1-LG19, according to [17].

\section{Phenotypic measurements}

The CPW was evaluated and the length of the third internode (IL) was measured at the end of 2009 and 2010. Shoot number per plant was two and the longest shoot was systematically chosen for measurements. Pearson correlation coefficients were evaluated using R [49].

\section{QTL analysis}

Data normality for each quantitative trait was evaluated with Shapiro-Wilkinson test. Despite deviations from normality for each trait, data were not transformed because the interval mapping method is robust to deviations from this assumption [50]. QTL detection was performed using the raw metric measurements with MapQTL 6.0 software [51] and adapted from the literature [9]. Four statistical methods were employed: Kruskal-Wallis analysis, interval mapping, MQM and permutation test. Four was retained as the maximum number of co-factors. The minimum LOD score used for QTLs detection was three. The significant LOD threshold was calculated at 5\% for the LG and for the genome-wide through 1000 permutations. The maximum LOD value was retained for QTL position and $\mathrm{a} \pm$ 2-LOD interval for the confidence interval. Differences between the genotype at the VVIV04, VVIN94 and VMC8B5 loci, and the shoot development traits were tested for significance using $\mathrm{R}$ [49] by applying analysis of variance (ANOVA) followed by Tukey test ( $p$ value $<$ 0.05).

\section{RNA extraction}

Young leaves and stems were harvested and immediately snap frozen in liquid nitrogen. Total RNA was extracted using the Spectrum Plant Total RNA kit (Sigma-Aldrich) according to the manufacturer's instructions.

\section{Microarray analysis}

Roche Nimblegen oligonucleotide microarrays (Design 090918 Vitus exp. HX12) were used for whole genome transcriptome analysis. This microarray probe design for the 29,549 transcripts studied is based on the $12 \mathrm{X}$ genome assembly using the grapevine V1 gene model prediction from CRIBI (http://genomes.cribi.unipd.it/). The correspondence between probe identifiers and gene identifiers were obtained from CRIBI V2 (http://genomes.cribi.unipd.it).

The microarray hybridisations were done by the Plateforme Biopuces, Institut National des Sciences Appliquées, Toulouse, France for the 10 individuals (five with a dwarfed and five with a normal phenotype); the protocol followed was as recommended by the manufacturer.

$\mathrm{R}$ was used to analyze the microarray data [49] as described by [52]. The limma package was used to identify differentially expressed genes [53]; genes with absolute $\log _{2}$ fold changes $>1$ and Holm corrected $p$ values below 0.05 were considered significant.

\section{qPCR analysis}

For qPCR experiments, total RNA was treated with the Turbo DNA-free kit from Ambion to remove genomic DNA contamination and the reverse transcription was done using the Superscript III kit from Invitrogen (using oligo dT primers and $1.5 \mu \mathrm{g}$ RNA). Gene expression was analyzed with iQ Sybr Green Supermix on a Biorad CFX96 machine (primer concentration of $250 \mathrm{nM}$ ). The expression of genes of interest was normalised with 
SAND protein (VIT_206s0004g02820) and one additional reference gene were used to confirm the stability of expression of VIT_206s0004g02820 (Additional file 10). Two technical replicates were used in this study. PCR efficiency for each primer pair was calculated using LinRegPCR [54].

\section{Plasmid DNA preparation and insert size estimation} DNA from CS bacterial artificial chromosome (BAC) clones VVCS1H006A20, VVCS1H011N07, VVCS1H012O11, VVC S1H12O17, VVCS1H018A11, VVCS1H065F12, VVC1H0 73F06 and VVCS1H03O10 [55] was isolated using the Nucleobond Xtra Midi Plus kit (Macherey Nagel) according to the manufacturer's instructions with chloramphenicol selective marker $\left(12.5 \mu \mathrm{g} \mathrm{mL}^{-1}\right)$. To estimate insert size, $150 \mathrm{ng}$ of each BAC was digested with the fast NotI enzyme (Fermentas) and incubated $40 \mathrm{~min}$ at $37^{\circ} \mathrm{C}$. After incubation, the enzymatic digestion was transferred in a gel (0.8\% agarose - TBE $0.25 \mathrm{X})$ for pulse field electrophoresis performed with a Chef Mapper XA CHILLER SYSTEM $220 \mathrm{~V}$ (Biorad) under the following conditions: voltage of $6 \mathrm{~V} \mathrm{~cm}^{-1}$, included angle of $120^{\circ}$, initial switch time of $5 \mathrm{~s}$, final switch time of $15 \mathrm{~s}$, run time of $16 \mathrm{~h}$ with linear ramping. Each insert size was estimated using the Genetools software (Syngene).

\section{Sanger sequencing of BAC extremities}

Based on estimated insert sizes, Sanger sequencing reactions were completed using Big Dye Terminator chemistry v3.1 (Applied Biosystems) on plasmid DNA of CS BAC clones VVCS1H018A11 and VVCS1H006A20 (around $300 \mathrm{ng}$ ) following the protocol described by [56] using T7 and M13r universal primers for BAC-end sequencing. Reaction products were analysed on an $\mathrm{ABI}$ 3730 DNA Analyzer (Applied Biosystems) at GeT-PlaGe platform (http://get.genotoul.fr/).

\section{PacBio sequencing}

About $1.5 \mu \mathrm{g}$ of DNA from both BACs VVCS1H018A11 and VVCS1H006A20 were pooled and sequenced using the standard Pacific Biosciences library preparation protocol for $10 \mathrm{~kb}$ libraries. Each replicate was sequenced in one SMRT Cell using the P6 polymerase in combination with the $\mathrm{C} 4$ chemistry, according to the manufacturer's instructions (by IGM: http://igm.ucsd. edu/genomics/).

\section{PacBio assembly}

Assembly of the PacBio reads was performed following the HGAP workflow (https://github.com/PacificBiosciences/Bioinformatics-Training/wiki/HGAP [57]. The $\mathrm{SMRT}^{\circ}$ Analysis (v2.3.0) software suite was used for HGAP implementation (https://github.com/PacificBiosciences/SMRT-Analysis).
Reads were first aligned by BLASR (https://github. com/PacificBiosciences/blasr; [58] against "Escherichia coli strain K12 substrain DH10B complete genome". Identified $E$. coli reads and low quality reads (read quality $<0.80$ and read length $<500 \mathrm{bp}$ ) were removed from data. Filtered reads were then preassembled to generate long sequences. The sequences obtained were filtered against vectors sequences and the Celera assembler was used to get a draft assembly. The last step of HGAP workflow was the "polishing" that significantly reduced the remaining insertions/deletions and base substitution errors in the draft assembly. The Quiver algorithm (https://github.com/PacificBiosciences/GenomicConsensus/blob/master/doc/QuiverFAQ.rst) was used to enrich the quality scores embedded in Pacific Biosciences bas.h5 files. The "polished assemblies" were identified by matching their BAC end sequences with BLAST.

\section{In silico analyses of BAC insert sequences}

The obtained insert sequences of BAC clones VVC S1H018A11 and VVCS1H006A20 were aligned to 12X.v2 genome sequence [59] using MUMmer4 software [60]; https://github.com/mummer4/mummer). The presence of interspersed repeats and low complexity DNA sequences was evaluated using the RepeatMasker2.1 software (https:// github.com/rmhubley/RepeatMasker).

\section{PCR-based deletion marker}

A pair of primers Delchr7F:5'-GGGTTGCAACTATG GTGATGCT-3' and Delchr7R: 5'-CACAGGCACGGGTC ACTCTC-3' were manually designed and used to detect the presence of the $84,482 \mathrm{bp}$ deletion in the genomic DNA of dwarfed genotypes of CSxRGM_F2 population and in the genome of $51 \mathrm{~V}$. vinifera cultivars (Additional file 9). All PCRs were performed in $15 \mu \mathrm{L}$ reaction volume containing: $10 \mathrm{ng}$ of template DNA, $1 \mathrm{x}$ PCR reaction buffer, $2 \mathrm{mM}$ $\mathrm{MgCl}_{2}, 0.2 \mathrm{mM}$ of each dNTP, $0.2 \mu \mathrm{M}$ of each primer, 0.025 $\mathrm{U}$ of JumpStart ${ }^{\mathrm{Tw}}$ Taq DNA Polymerase (Sigma Aldrich). The PCR thermocycler conditions were $5 \mathrm{~min}$ initial denaturation step at $94^{\circ} \mathrm{C}$ followed by 35 cycles of $30 \mathrm{~s}$ denaturation at $94{ }^{\circ} \mathrm{C}, 1 \mathrm{~min}$ annealing at $62^{\circ} \mathrm{C}$ and $1 \mathrm{~min}$ extension at $72{ }^{\circ} \mathrm{C}$, followed by $5 \mathrm{~min}$ final extension at $72^{\circ} \mathrm{C}$. The PCR product obtained were analysed on $1.8 \%$ agarose gel. A PCR product length of $782 \mathrm{bp}$ is observed when a deletion is present in the genome of evaluated genotypes.

\section{Supplementary information}

Supplementary information accompanies this paper at https://doi.org/10. 1186/s12870-020-2258-0.

Additional file 1: Table S1. New SSR markers developed

Additional file 2: Figure S1. Linkage map of Vitis vinifera cv. CabernetSauvignon $\times$ V. riparia $\mathrm{CV}$. riparia Gloire de Montpellier F2 population 
(CS x RGM F2). Linkage groups are named according to international consensus map. Distances are in cM Kosambi

Additional file 3: Table S2. Characteristics of the CS x RGM_F2 linkage map

Additional file 4: Figure S2. Photographs of CS $\times$ RGM F2 population growing in greenhouse. a Genotypes with normal phenotypes. $\mathbf{b}$-d Individuals with dwarfed phenotypes showing curled leaves $(\mathbf{e}-\mathbf{g})$

Additional file 5: Figure S3. Validation of microarray data by $\mathrm{QPCR}$ of genes differentially expressed between dwarfed and normal individuals from CS x RGM_F2 population. a VIT_20750031g00320; b VIT_20750031g00330; c VIT_207s0031g00340; d VIT_207s0031g00350; e VIT_214s0108g00810 and f VIT_21450108g00760. Means and standard errors shown, $n=3$

Additional file 6: Table S3. Alignment of WCS1H006A2O insert BAC sequence to the chromosome 7 sequence. MUMmer4 software was used to align insert BAC sequence to the chromosome 7 sequence from the 12X.v2 genome

Additional file 7: Table S4. Alignment of WCS1H018A11 insert BAC sequence to the chromosome 7 sequence. MUMmer4 software was used to align insert BAC sequence to the chromosome 7 sequence from the 12X.v2 genome

Additional file 8: Figure S4. The physical map of the $84,482 \mathrm{bp}$ deletion on chromosome 7 based on the BAC clone WCS1H018A11 Double arrows delimited the two large deletions identified of 13,084 bp and 84,482 bp respectively. All dotted lines delimited similar sequences between the chromosome 7 sequence from the 12X.v2 PN40024 reference genome and the BAC clone WVCS1H018A11 (Additional file 7). Red dotted lines were used for transposition-inversions and green ones for transpositions. Physical positions are given in kb for the BAC clone WCS1H018A11 sequence and in Mb for the chromosome 7 of PN40024 genotype. The 11 genes deleted on chromosome 7 of the dwarfed plants are symbolized in blue

Additional file 9: Table S5. Presence of the deletion within the genome of fifty-one grapevine cultivars. -, absence of deletion. +, presence of deletion

Additional file 10: Table S6. Sequence and mean PCR efficiency of primers used for $\mathrm{QPCR}$ analysis

\section{Abbreviations}

BAC: Bacterial artificial chromosome; CM: Centimorgan; CPW: Winter cane pruning weight; CS: Vitis vinifera cv. Cabernet-Sauvignon; Cv.: Cultivar; IL: Internode length; LG: Linkage group; LOD: Logarithm of the odds; MQM: Multiple QTL mapping; QTL: Quantitative Trait Loci; RGM: Vitis riparia CV. riparia Gloire de Montpellier; SSR: Simple sequence repeat

\section{Acknowledgements}

We thank Christophe Boury, Agnès Destrac, Martine Donnart, Bernard Douens, Virginie Garcia, Nabil Girollet, Cyril Hévin, Ghislaine Hilbert, Elena Morin, Jean-Pierre Petit and Jean-Paul Robert for their technical assistance. We thank the Genome Transcriptome Facility of Bordeaux (PGTB) for sequencing of microsatellites (Pierroton, France), the Plateforme Biopuces (Institut National des Sciences Appliquées, Toulouse, France) for microarray hybridisations, the INRA-CNRGV (French Plant Genomic Resource Center, CastanetTolosan, France) for the use of BAC sequences and the GeT-PlaGe platform for BACs sequencing (Toulouse, France).

\section{Availability of data and materials}

The CS X RGM_F2 map is available with new SSR markers on https://doi.org/ $10.15454 / 4 Z$ UPCP. The raw and normalised microarray data is available on http://www.ebi.ac.uk/arrayexpress; the accession number is E-MTAB-2472. BAC insert sequences are available on https://doi.org/10.15454/4ZUPCP.

\section{Authors' contributions}

SG and StD carried out the analysis for genetic map construction, conducted measurements on the CS $\times$ RGM F2 population and performed the QTL detection. SG identified the deletion and defined the genetic marker related. SJC studied genes expression by quantitative PCR and microarray analysis. EG and SJC coordinated the project. SG, EG and SJC wrote the manuscript.
NO and SD substantively revised it. All authors reviewed, edited and approved the final manuscript.

\section{Funding}

This work was supported by The Agence Nationale pour la Recherche (grant ANR-09-GENM-023-002 VITAROMA) and by the Comité National des Interprofessions des Vins. The funding bodies had no role in the design of the study and collection, analysis, and interpretation of data and in writing the manuscript.

Ethics approval and consent to participate

Not applicable.

\section{Consent for publication}

Not applicable.

\section{Competing interests}

The authors declare that they have no competing interests.

\section{Author details}

'UMR1287 EGFV, Bordeaux Sciences Agro, INRAE, University of Bordeaux, Villenave d'Ornon, France. ${ }^{2}$ UMR1332 BFP, INRAE, University of Bordeaux, Villenave d'Ornon, France.

Received: 6 July 2019 Accepted: 20 January 2020

Published online: 29 January 2020

\section{References}

1. Keller M. The science of grapevines : anatomy and physiology. 1st ed. London: Elsevier; 2010

2. Townsley BT, Sinha NR. A new development: evolving concepts in leaf ontogeny. Annu Rev Plant Biol. 2012;63:535-62.

3. Boss PK, Thomas MR. Association of dwarfism and floral induction with a grape 'green revolution' mutation. Nature. 2002;416:847-50.

4. Chaib J, Torregrosa L, Mackenzie D, Corena P, Bouquet A, Thomas MR. The grape microvine - a model system for rapid forward and reverse genetics of grapevines. Plant J. 2010;62:1083-92.

5. Houel C, Chatbanyong R, Doligez A, Rienth M, Foria S, Luchaire N, Roux C, Adiveze A, Lopez G, Farnos M, et al. Identification of stable QTLs for vegetative and reproductive traits in the microvine (Vitis vinifera $L$.) using the $18 \mathrm{~K}$ Infinium chip. BMC Plant Biol. 2015;15:205.

6. Filler DM, Luby JJ, Ascher PD. Incongruity in the interspecific crosses of Vitis $L$ reproductive expression in the $F 1$ progeny. Euphytica. 1994:78:155-64.

7. Filler DM, Luby JJ, Ascher PD. Incongruity in the interspecific crosses of Vitis L morphological abnormalities in the F2 progeny. Euphytica. 1994;78:227-37.

8. Welter JW, Grando MS, Zyprian E. Basics of grapevine genetic analysis. Enfield: Science Publishers; 2011.

9. Marguerit E, Boury C, Manicki A, Donnart M, Butterlin G, Nemorin A, Wiedemann-Merdinoglu S, Merdinoglu D, Ollat N, Decroocg S. Genetic dissection of sex determinism, inflorescence morphology and downy mildew resistance in grapevine. Theor Appl Genet. 2009;118:1261-78.

10. Chen J, Wang N, Fang LC, Liang ZC, Li SH, Wu BH. Construction of a highdensity genetic map and QTLs mapping for sugars and acids in grape berries. BMC Plant Biol. 2015:15:28.

11. Yang S, Fresnedo-Ramírez J, Sun Q, Manns DC, Sacks GL, Mansfield AK, Luby JJ, Londo JP, Reisch Bl, Cadle-Davidson LE, et al. Next generation mapping of enological traits in an F2 interspecific grapevine hybrid family. PLoS One. 2016. https://doi.org/10.1371/journal.pone.0149560.

12. Fechter I, Hausmann L, Zyprian E, Daum M, Holtgrawe D, Weisshaar B, Topfer R. QTL analysis of flowering time and ripening traits suggests an impact of a genomic region on linkage group 1 in Vitis. Theor Appl Genet. 2014;127:1857-72

13. Duchene E, Butterlin G, Dumas V, Merdinoglu D. Towards the adaptation of grapevine varieties to climate change: QTLs and candidate genes for developmental stages. Theor Appl Genet. 2012;124:623-35.

14. Zhao YH, Su K, Guo YH, Ma HF, Guo XW. Molecular genetic map construction and QTL analysis of fruit maturation period in grapevine. Genet Mol Res. 2016. https://doi.org/10.4238/gmr.15028040.

15. Zyprian E, Ochssner I, Schwander F, Simon S, Hausmann L, Bonow-Rex M, Moreno-Sanz P, Grando MS, Wiedemann-Merdinoglu S, Merdinoglu D, et al. 
Quantitative trait loci affecting pathogen resistance and ripening of grapevines. Mol Gen Genomics. 2016;291:1573-94.

16. Cipriani G, Di Gaspero G, Canaguier A, Jusseaume J, Tassin J, Lemainque A, Thareau V, Adam-Blondon AF, Testolin R. Molecular linkage maps: strategies, resources and achievements. In: Adam-Blondon AF, Martinez-Zapater JM, Kole C, editors. Genetics, genomics and breeding in grapes. Enfield: Science Publishers; 2011. p. 111-36.

17. Adam-Blondon AF, Roux C, Claux D, Butterlin G, Merdinoglu D, This P. Mapping 245 SSR markers on the Vitis vinifera genome: a tool for grape genetics. Theor Appl Genet. 2004;109:1017-27.

18. Hvarleva TD, Russanov KE, Bakalova AT, Zhiponova MK, Djakova GJ, Atanassov Al, Atanassov II. Microsatellite linkage map based on F2 population from Bulgarian grapevine cultivar Storgozia. Biotechnol Biotechnol Equip. 2009;23:1126-30.

19. Dunlevy JD, Dennis EG, Soole KL, Perkins MV, Davies C, Boss PK. A methyltransferase essential for the methoxypyrazine-derived flavour of wine. Plant J. 2013:75:606-17.

20. Van Ooijen JW, Jansen J. Genetic mapping in experimental populations. Cambridge: Cambridge University Press; 2013

21. Li YH, Yang LM, Pathak M, Li DW, He XM, Weng YQ. Fine genetic mapping of $\mathrm{cp}$ : a recessive gene for compact (dwarf) plant architecture in cucumber, Cucumis sativus L. Theor Appl Genet. 2011;123:973-83.

22. Fukuta N, Fujioka S, Takatsuto S, Yoshida S, Fukuta Y, Nakayama M. 'Rinrei', a brassinosteroid-deficient dwarf mutant of faba bean (Vicia faba). Physiol Plant. 2004;121:506-12.

23. Li ZF, Guo Y, Ou L, Hong HL, Wang J, Liu ZX, Guo BF, Zhang L, Qiu L. Identification of the dwarf gene GmDW1 in soybean (Glycine max L.) by combining mapping-by-sequencing and linkage analysis. Theor Appl Genet. 2018;131:1001-16.

24. Lawit SJ, Wych HM, Xu DP, Kundu S, Tomes DT. Maize DELLA proteins dwarf plant8 and dwarf plant9 as modulators of plant development. Plant Cell Physiol. 2010;51:1854-68.

25. Jiang F, Guo M, Yang F, Duncan K, Jackson D, Rafalski A, Wang S, Li B. Mutations in an AP2 transcription factor-like gene affect internode length and leaf shape in maize. PLoS One. 2012. https:/doi.org/10.1371/journal.pone.0037040.

26. Lopez-Vernaza M, Yang SX, Muller R, Thorpe F, de Leau E, Goodrich J. Antagonistic roles of SEPALLATA3, FT and FLC genes as targets of the Polycomb group gene CURLY LEAF. PLoS One. 2012. https://doi.org/10. 1371/journal.pone.0030715.

27. Goodrich J, Puangsomlee P, Martin M, Long D, Meyerowitz EM, Coupland G A polycomb-group gene regulates homeotic gene expression in Arabidopsis. Nature. 1997:386:44-51.

28. Gomez-Mena C, de Folter S, Costa MMR, Angenent GC, Sablowski R. Transcriptional program controlled by the floral homeotic gene AGAMOUS during early organogenesis. Development. 2005;132:429-38.

29. Almada R, Cabrera N, Casaretto JA, Pena-Cortes H, Ruiz-Lara S, Villanueva EG Epigenetic repressor-like genes are differentially regulated during grapevine (Vitis vinifera L.) development. Plant Cell Rep. 2011;30:1959-68.

30. Dowd PE, Gilroy $\mathrm{S}$. The emerging roles of phospholipase $\mathrm{C}$ in plant growth and development. Lipid Signal Plants Plant Cell Monogr. 2010;16:23-37.

31. Sadanandom A, Bailey M, Ewan R, Lee J, Nelis S. The ubiquitin-proteasome system: central modifier of plant signalling. New Phytol. 2012;196:13-28.

32. Yang XH, Kalluri UC, Jawdy S, Gunter LE, Yin TM, Tschaplinski TJ, Weston DJ, Ranjan $P$, Tuskan GA. The F-box gene family is expanded in herbaceous annual plants relative to Woody perennial plants. Plant Physiol. 2008;148:1189-200.

33. Kumar M, Campbell L, Turner S. Secondary cell walls: biosynthesis and manipulation. J Exp Bot. 2016;67:515-31.

34. Do CT, Pollet B, Thevenin J, Sibout R, Denoue D, Barriere Y, Lapierre C, Jouanin L. Both caffeoyl coenzyme a 3-O-methyltransferase 1 and caffeic acid Omethyltransferase 1 are involved in redundant functions for lignin, flavonoids and sinapoyl malate biosynthesis in Arabidopsis. Planta. 2007;226:1117-29.

35. Zhong RQ, Morrison WH, Himmelsbach DS, Poole FL, Ye ZH. Essential role of caffeoyl coenzyme a O-methyltransferase in lignin biosynthesis in woody poplar plants. Plant Physiol. 2000;124:563-77.

36. Vanholme R, Storme V, Vanholme B, Sundin L, Christensen JH, Goeminne G, Halpin C, Rohde A, Morreel K, Boerjan W. A systems biology view of responses to lignin biosynthesis perturbations in Arabidopsis. Plant Cell. 2012;24:3506-29

37. Hu W, Ma H. Characterization of a novel putative zinc finger gene MIF1: involvement in multiple hormonal regulation of Arabidopsis development. Plant J. 2006;45:399-422.
38. Hu W, Feng BM, Ma H. Ectopic expression of the Arabidopsis MINI ZINC FINGER1 and MIF3 genes induces shoot meristems on leaf margins. Plant Mol Biol. 2011;76:57-68.

39. Girollet N, Rubio B, Lopez-Roques C, Valière S, Ollat N, Bert PF. De novo phased assembly of the Vitis riparia grape genome. Sci Data. 2019;6:127.

40. Myles S, Boyko AR, Owens CL, Brown PJ, Grassi F, Aradhya MK, Prins B, Reynolds A, Chia JM, Ware D, et al. Genetic structure and domestication history of the grape. Proc Natl Acad Sci U S A. 2011;108:3530-5.

41. Robinson J, Harding J, Vouillamoz J. Wine grapes. A complete guide to 1,368 vine varieties, including their origin and flavours. London: Penguin Books Ltd; 2012.

42. Lacombe T, Boursiquot JM, Laucou V, Di Vecchi-Staraz M, Peros JP, This P. Large-scale parentage analysis in an extended set of grapevine cultivars (Vitis vinifera L.). Theor Appl Genet. 2013;126:401-14.

43. Sahebi M, Hanafi MM, van Wijnen AJ, Rice D, Raffi MY, Azizi P, Osman M, Taheri S, Abu Bakar MF, Isa MNM, et al. Contribution of transposable elements in the plant's genome. Gene. 2018;665:155-66.

44. Mehrotra S, Goyal V. Repetitive sequences in plant nuclear DNA: types, distribution, evolution and function. Genomics Proteomics Bioinform. 2014;12:164-71.

45. Garrido-Ramos MA. Satellite DNA in Plants: More than Just Rubbish. Cytogenet Genome Res. 2015;146:153-70.

46. Guillaumie S, llg A, Rety S, Brette M, Trossat-Magnin C, Decroocq S, Leon C, Keime $C$, Ye T, Baltenweck-Guyot R, et al. Genetic analysis of the biosynthesis of 2-Methoxy-3-Isobutylpyrazine, a major grape-derived aroma compound impacting wine quality. Plant Physiol. 2013;162:604-15.

47. de Givry S, Bouchez M, Chabrier P, Milan D, Schiex T. CARTHAGENE: multipopulation integrated genetic and radiation hybrid mapping. Bioinformatics. 2005;21:1703-4.

48. Stam P. Construction of integrated genetic-linkage maps by means of a new computer package - JoinMap. Plant J. 1993;3:739-44.

49. R Core Team. R: A language and environment for statistical computing. In: R Foundation for Statistical Computing. 2017. http://www.R-project.org. Accessed 10 April 2017.

50. Rebai A. Comparison of methods for regression interval mapping in QTL analysis with non-normal traits. Genet Res. 1997;69:69-74.

51. van Ooijen JW, Boer MP, Jansen RC, Maliepaard CA. MapQTL 4.0: Software for the calculation of QTL positions on genetic maps (user manual). In: Wageningen: Plant Research International; 2000.

52. Cookson SJ, Ollat N. Grafting with rootstocks induces extensive transcriptional re-programming in the shoot apical meristem of grapevine. BMC Plant Biol. 2013;13:147.

53. Smyth GK. Limma: linear models for microarray data. In: Gentleman R, Carey V, Dudoit S, Irizarry R, W H, editors. Bioinformatics and Computational Biology Solutions using R and Bioconductor. New York: Springer; 2005. p. 397-420.

54. Ramakers C, Ruijter JM, Deprez RHL, Moorman AFM. Assumption-free analysis of quantitative real-time polymerase chain reaction (PCR) data. Neurosci Lett. 2003;339:62-6.

55. Lamoureux D, Bernole A, Le Clainche I, Tual S, Thareau V, Paillard S, Legeai $F$, Dossat $C$, Wincker $P$, Oswald $M$, et al. Anchoring of a large set of markers onto a BAC library for the development of a draft physical map of the grapevine genome. Theor Appl Genet. 2006;113:344-56.

56. Santos AA, Penha HA, Bellec A, CdF M, Pedrosa-Harand A, Berges H, Carneiro Vieira ML. Begin at the beginning: a BAC-end view of the passion fruit (Passiflora) genome. BMC Genomics. 2014;15:816.

57. Chin CS, Alexander DH, Marks P, Klammer AA, Drake J, Heiner C, Clum A, Copeland A, Huddleston J, Eichler EE, et al. Nonhybrid, finished microbial genome assemblies from long-read SMRT sequencing data. Nat Methods. 2013;10:563-9.

58. Chaisson MJ, Tesler G. Mapping single molecule sequencing reads using basic local alignment with successive refinement (BLASR): application and theory. BMC Bioinform. 2012;13:238.

59. Canaguier A, Grimplet J, Di Gaspero G, Scalabrin S, Duchene E, Choisne N, Mohellibi N, Guichard C, Rombauts S, Le Clainche I, et al. A new version of the grapevine reference genome assembly (12X.v2) and of its annotation (VCost.v3). Genomics Data. 2017;14:56-62.

60. Marcais G, Delcher AL, Phillippy AM, Coston R, Salzberg SL, Zimin A. MUMmer4: A fast and versatile genome alignment system. PLoS Comput Biol. 2018. https://doi.org/10.1371/journal.pcbi.1005944.

\section{Publisher's Note}

Springer Nature remains neutral with regard to jurisdictional claims in published maps and institutional affiliations. 